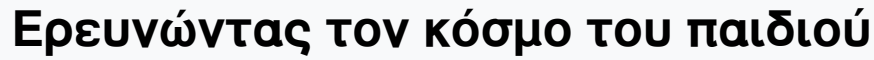

Tóp. 6 (2005)

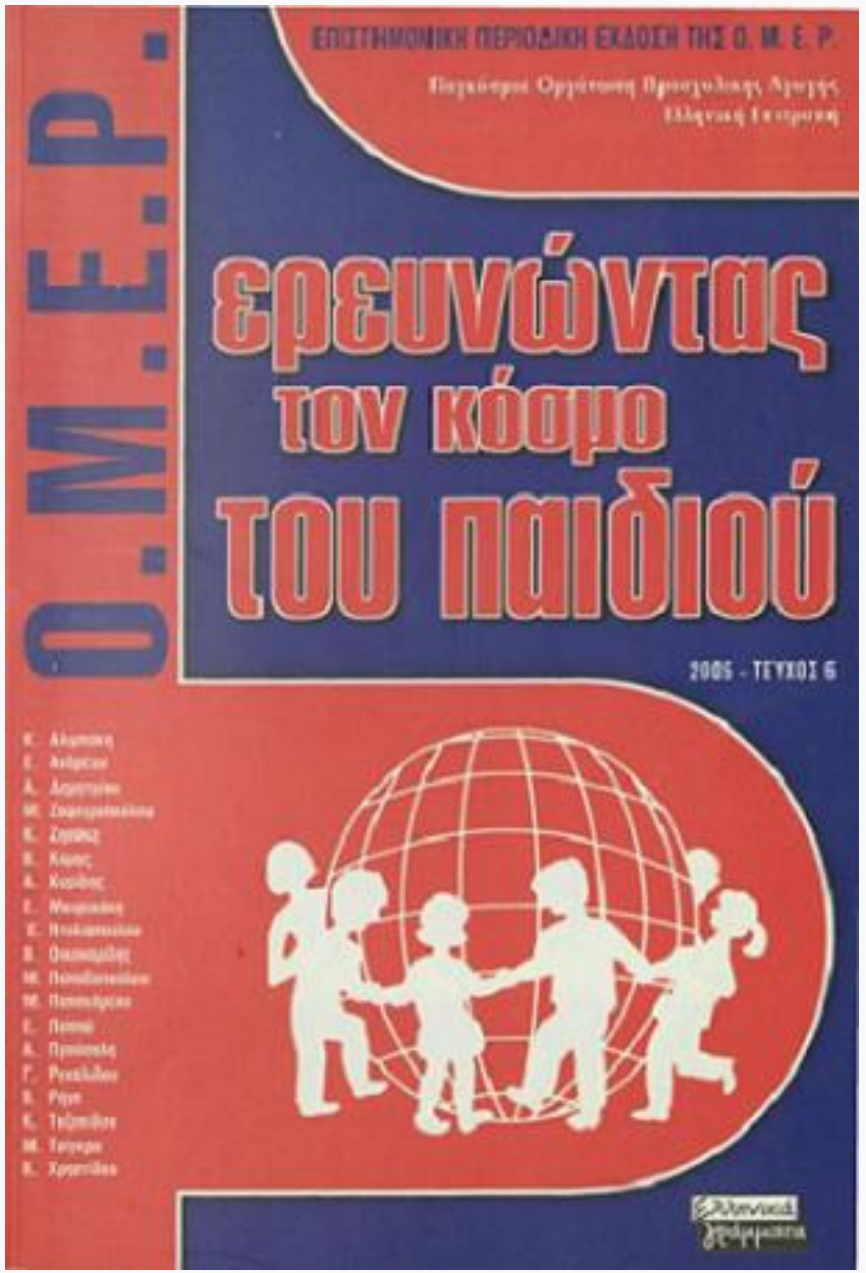

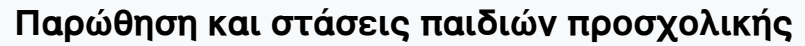

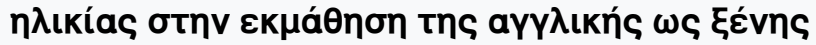

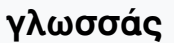

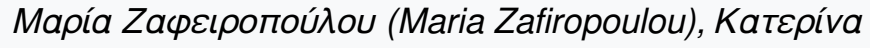

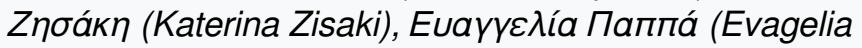
Papa)

doi: $10.12681 /$ icw.18401

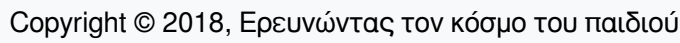

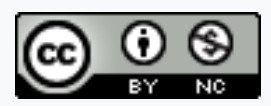

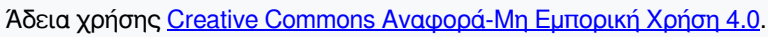

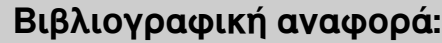

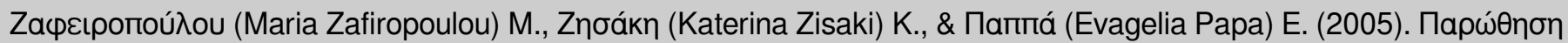

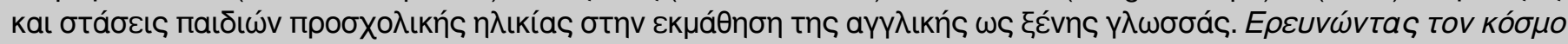
тоu паıઠıои́, 6, 44-58. https://doi.org/10.12681/icw.18401 


\section{Mapía Zaфєıропоúlou}

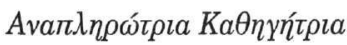

Iuxoloyías

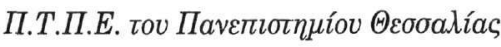

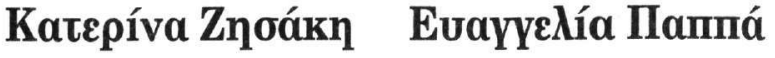

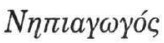

$\Delta \imath \delta a ́ \kappa \tau \omega \rho ~ \Pi a v \varepsilon \pi \imath \sigma \eta \mu i ́ o v$

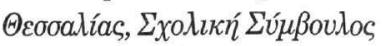

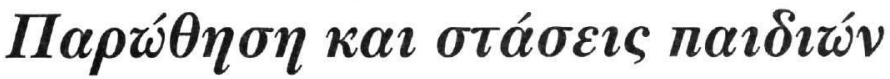

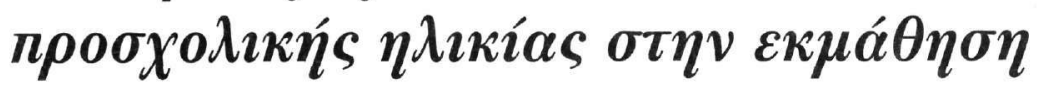

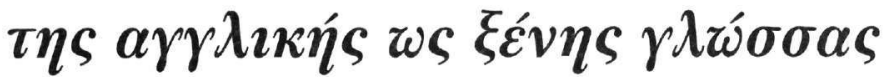

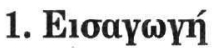

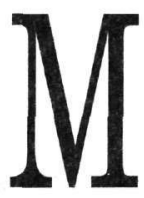

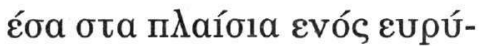

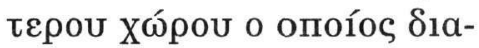
крívetal aпó tᄁv паүко-

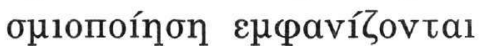

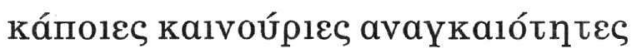

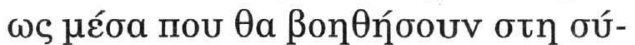

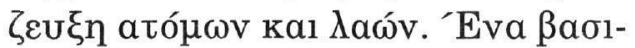

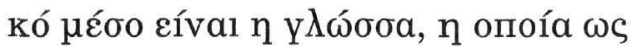

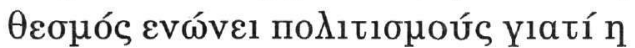

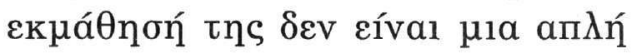

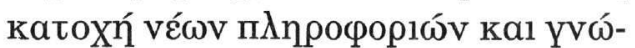

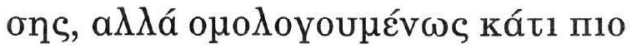
oúv $\theta \varepsilon \tau$.

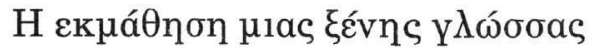

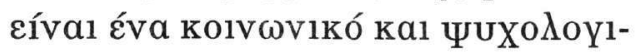
kó qaivópevo. Kal au tó Yiatí ótav

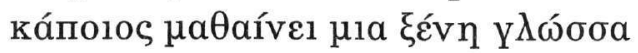

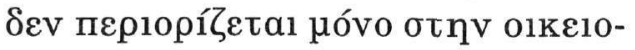

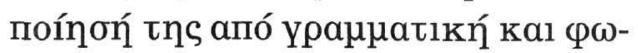

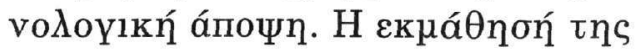
бUvenáYetal kal tᄁv пробéүYion

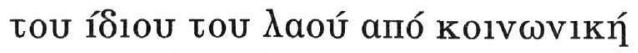

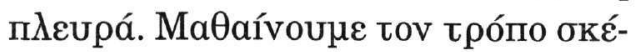

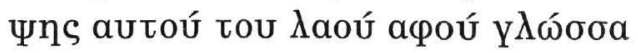

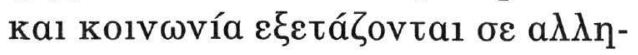

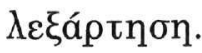

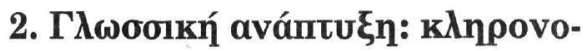

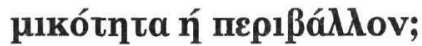

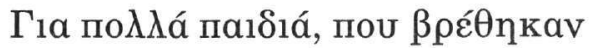

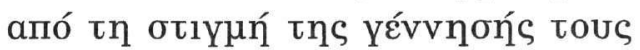

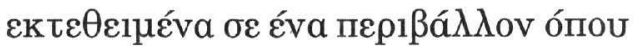

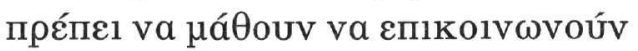

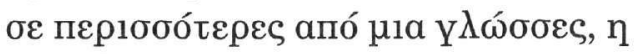

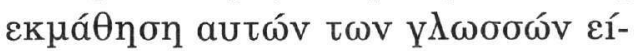
val kát1 aпóduta quб1одоү1кó ка1

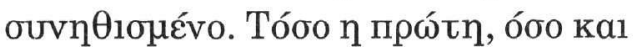

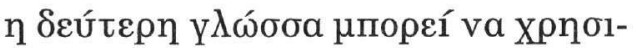

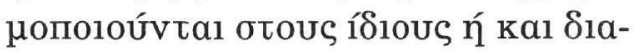

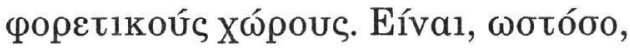

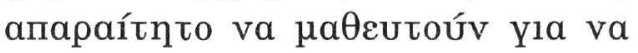

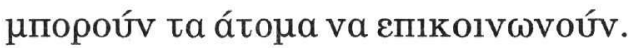

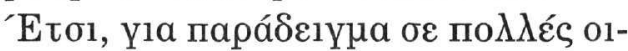

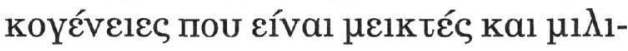

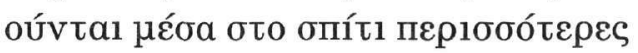

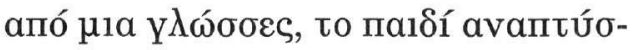

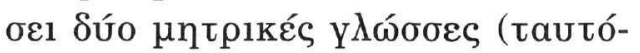

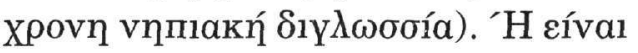

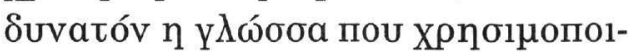

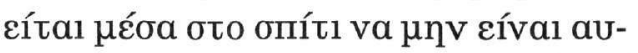

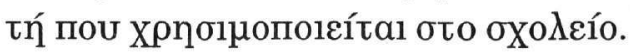

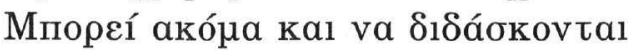

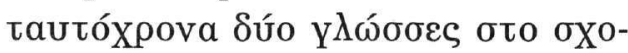

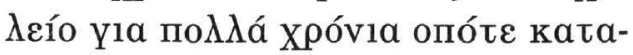




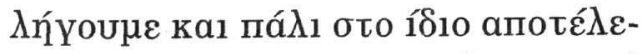

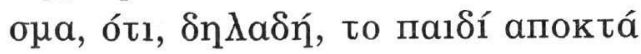

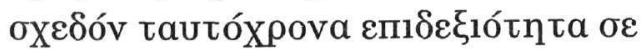

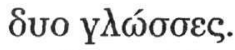

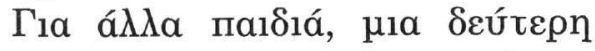

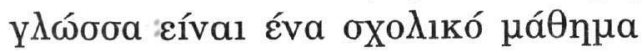

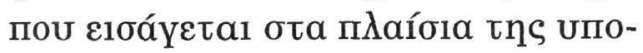

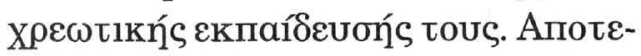

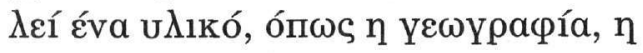

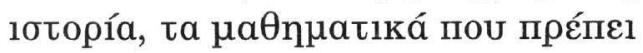
va $\delta 1 \delta a X \theta \varepsilon i ́$, va katavon $\theta \varepsilon i ́$, va $\mu a-$

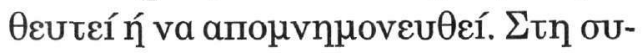

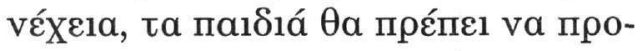

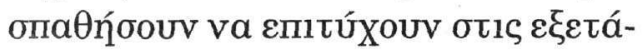

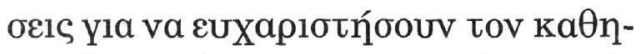

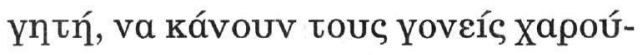

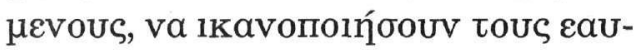

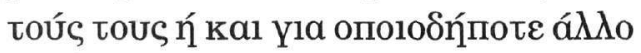

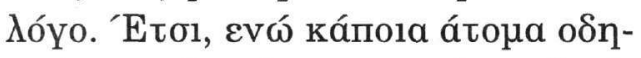

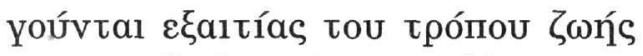

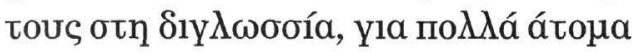

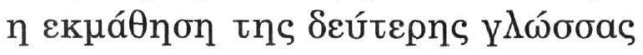

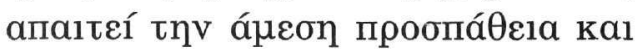

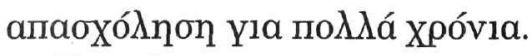

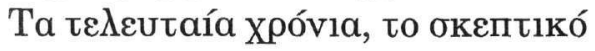

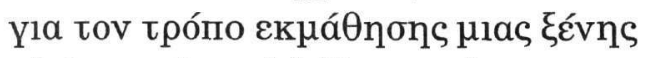

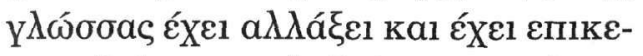

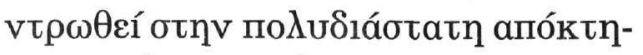

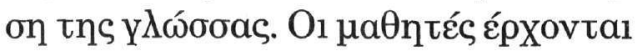

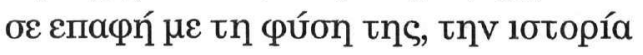

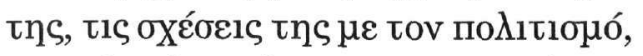

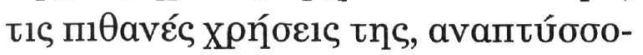

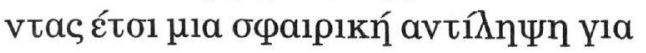

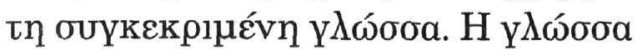

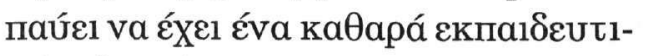

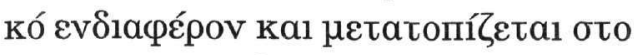
ке́v

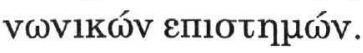

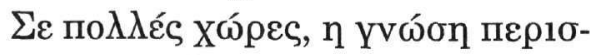

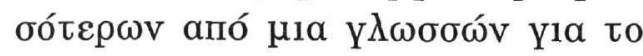

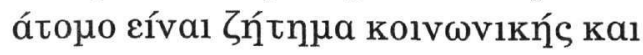
olkovouıñ́s emißíwons.

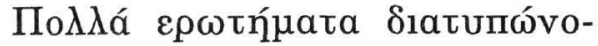

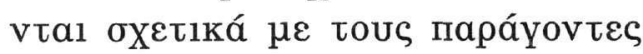

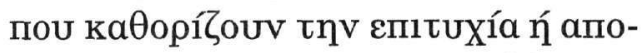

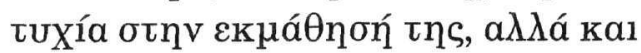

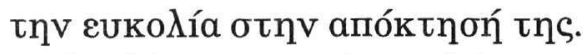

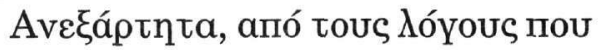

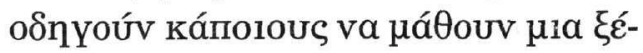

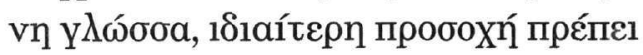

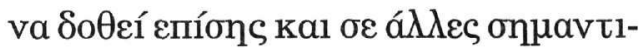

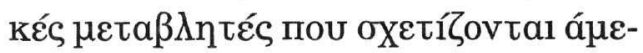

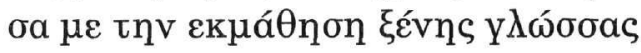

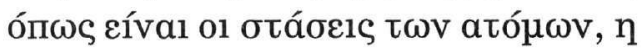

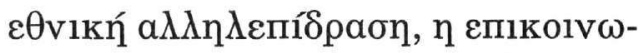

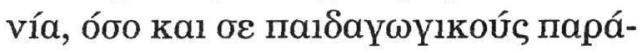

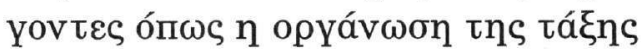

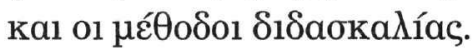

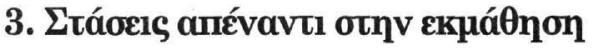

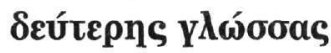

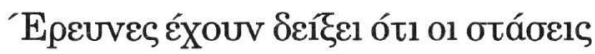

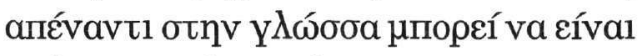

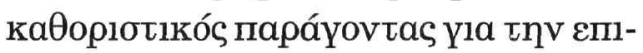

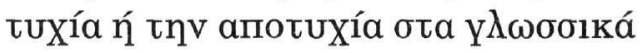

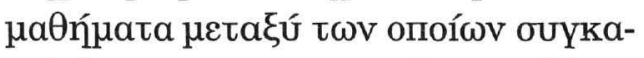

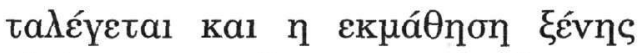
y

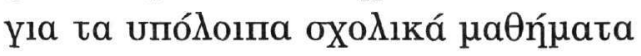
(Clement \& Kruidenier, 1985). O1 otá-

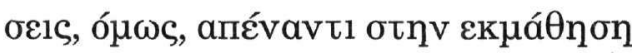

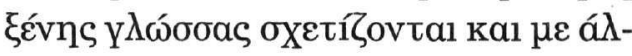

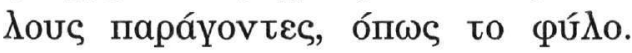

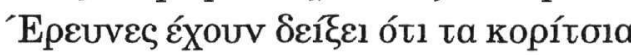

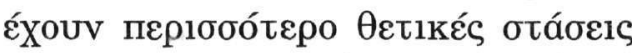

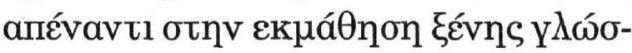

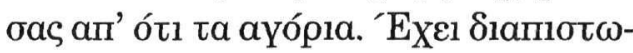

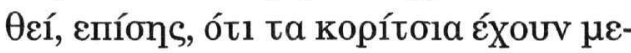

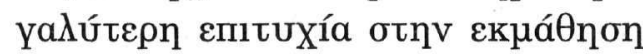

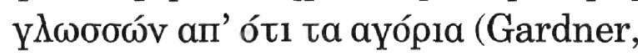

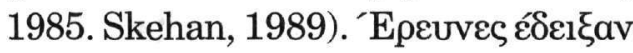

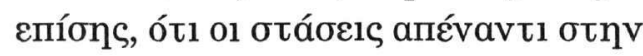




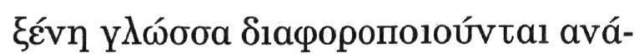

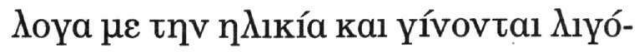

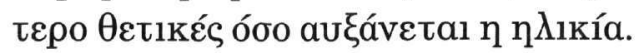

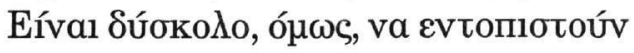

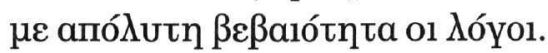

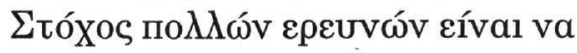

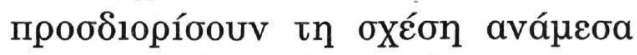

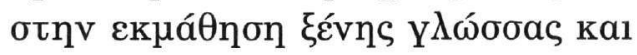

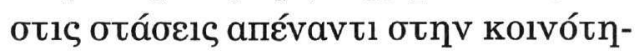

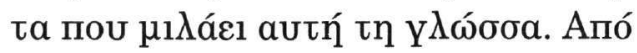
tous прштопópous autŕs tns про-

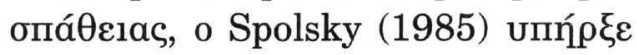

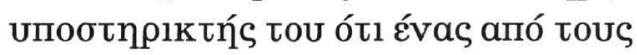

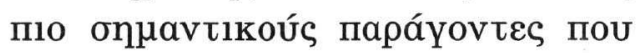

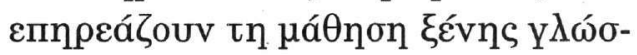

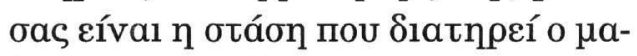

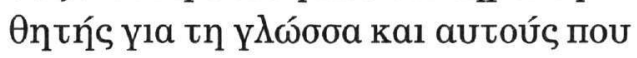
เท $\mu_{1} \lambda$ áve.

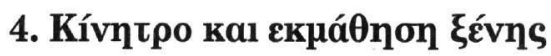

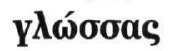

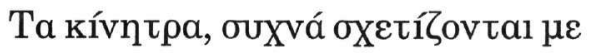

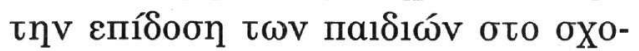

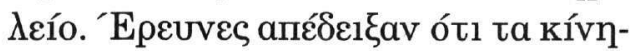

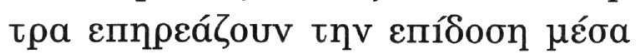

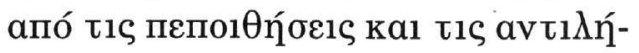

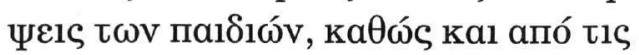

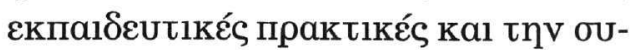

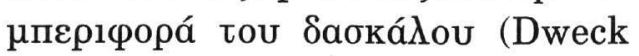

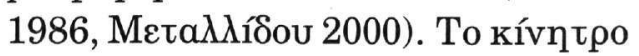

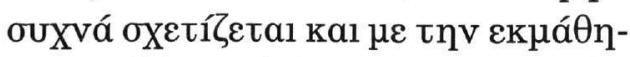

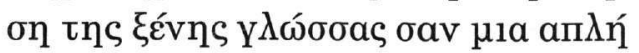

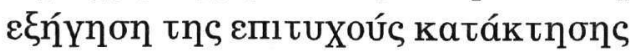

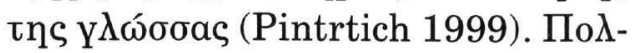

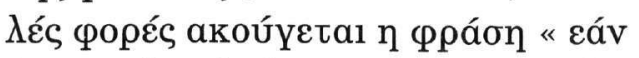

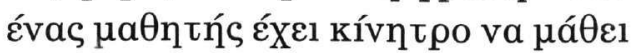

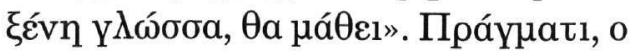

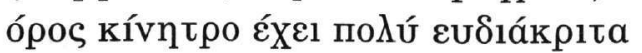

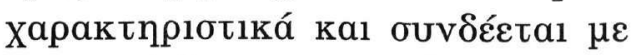

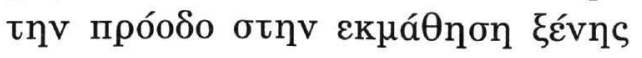

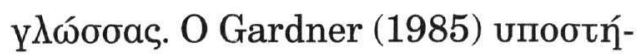

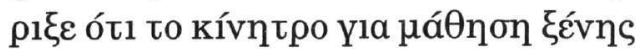
y

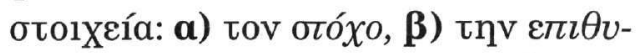

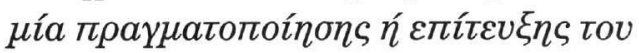

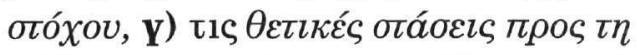

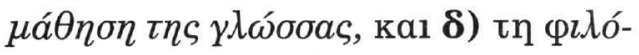

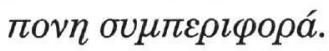

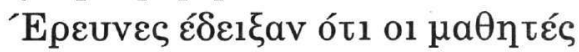

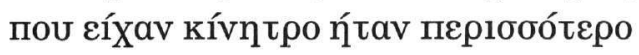

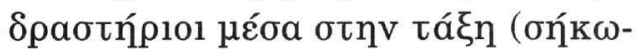

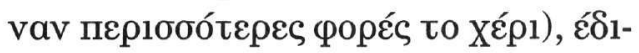

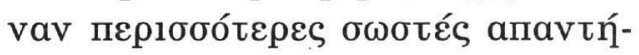

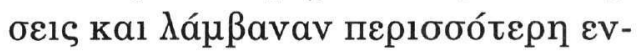

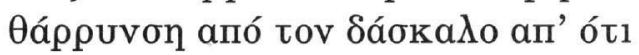

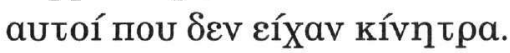

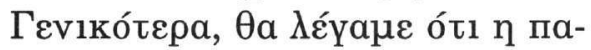

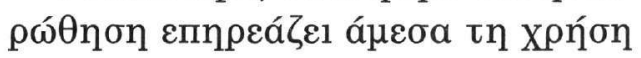

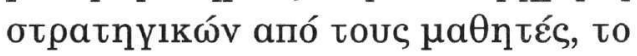

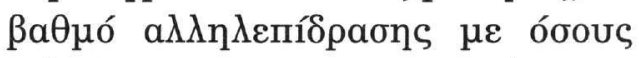
$\mu 1 \lambda$ oúv

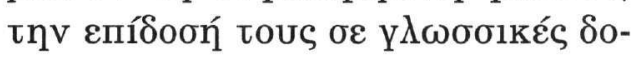

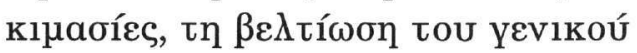

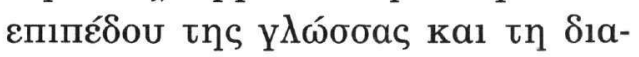

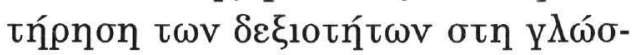

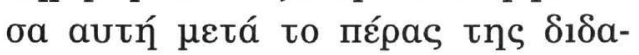

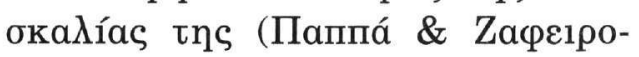
поúlou, 2000).

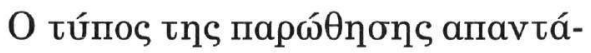

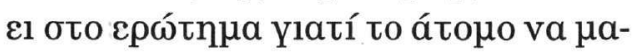

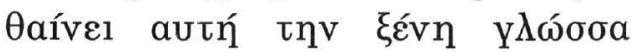
(Oxford \& Shearin, 1994. Gardner,

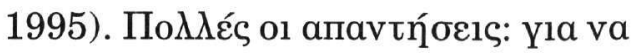

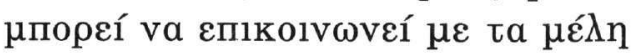

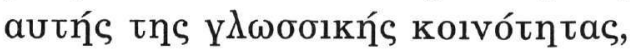

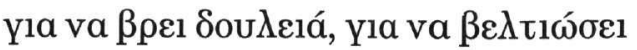

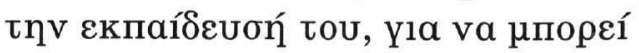

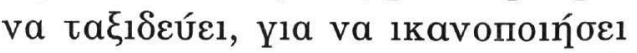

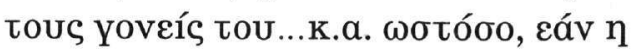

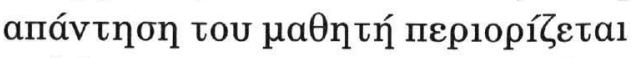

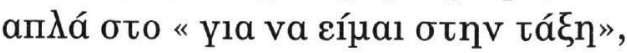

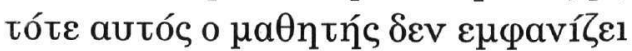




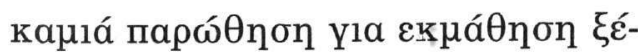

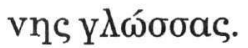

O1 Gardner kal Lambert (1972)

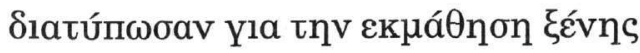

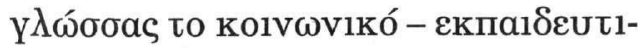

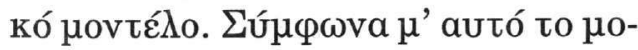

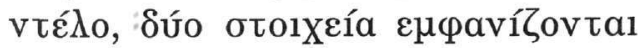

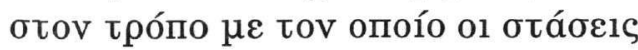

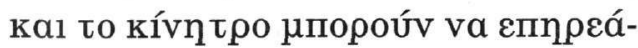

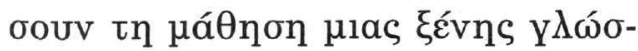

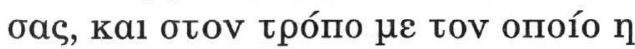

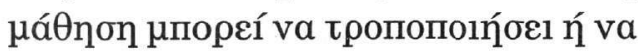

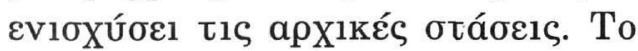

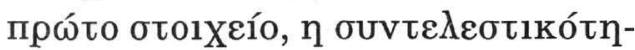

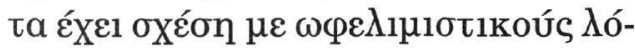

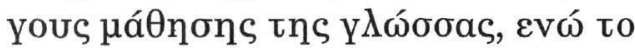

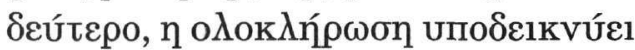

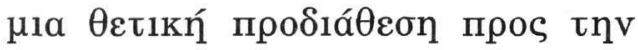

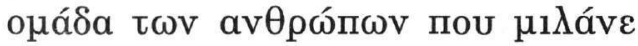

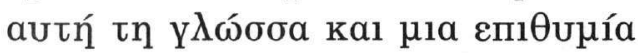

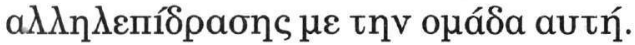

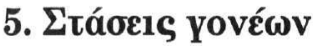

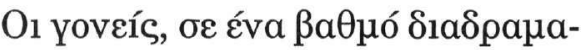

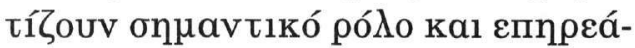

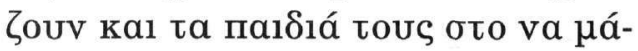

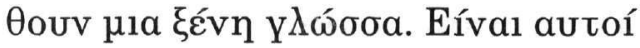

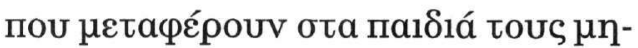

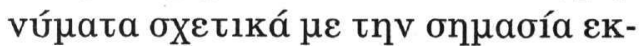

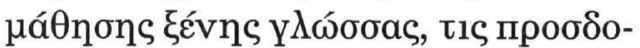
kírc touৎ nou a

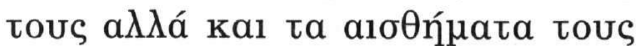

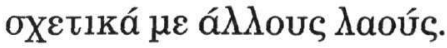

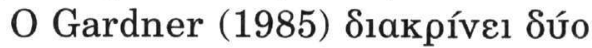

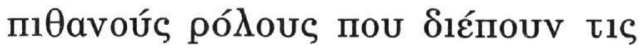

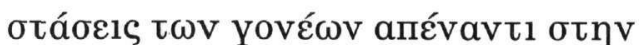

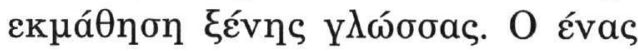

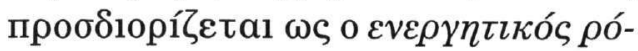
los kal avtıkatont

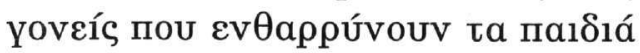

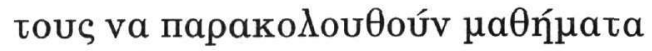

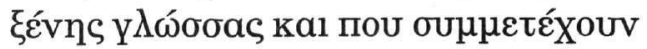

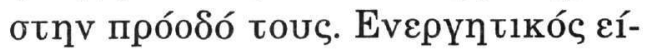

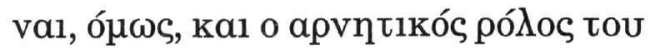

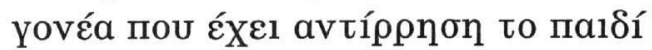

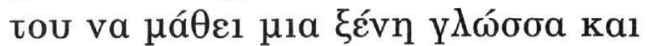

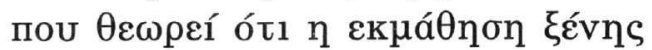

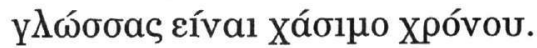

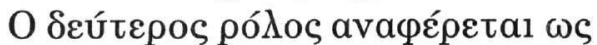

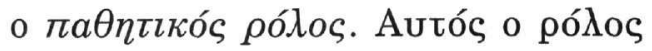

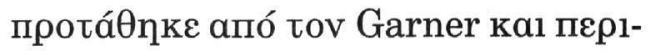

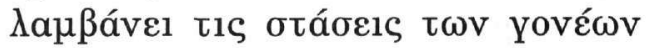

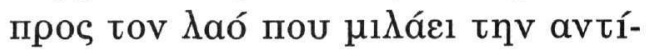

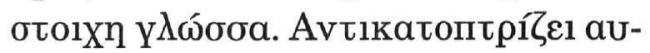

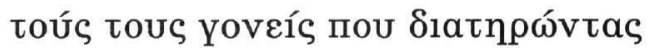

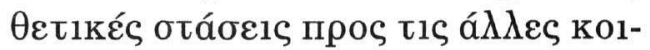

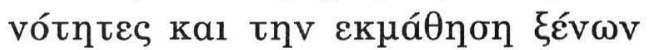

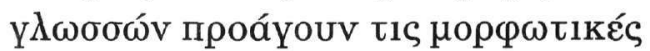

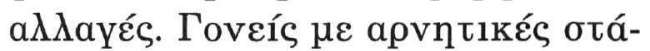

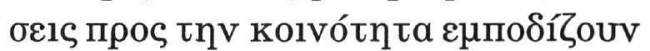

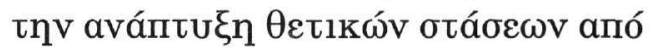

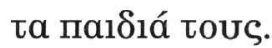

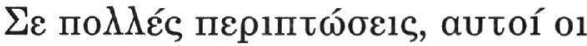

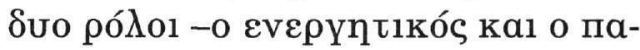

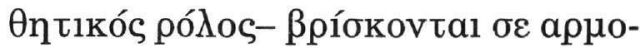

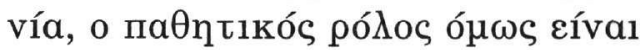

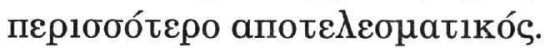

Oı yoveís anoteloúv to onja-

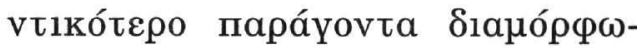

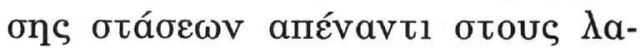

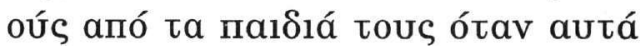

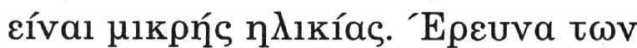
Lambert kal Klineberg (1967) ano-

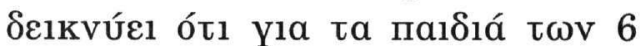

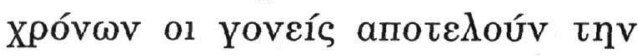

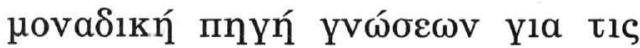

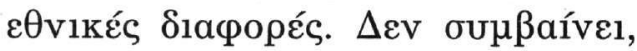

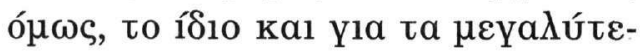

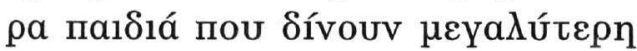

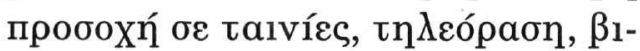

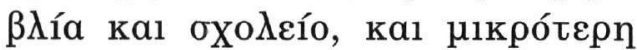

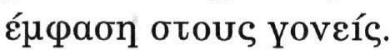




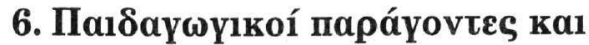

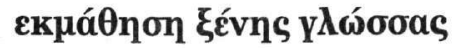

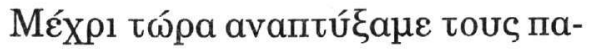

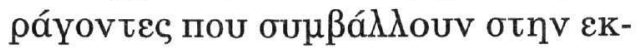

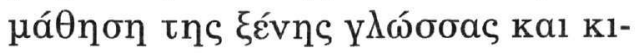

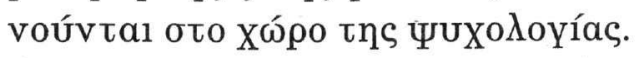

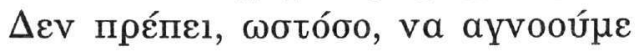

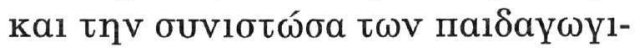

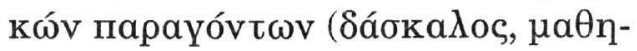

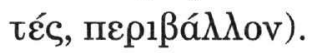

$\Sigma$ úp $\varphi$ vva $\mu \varepsilon$ tous Glover ка1

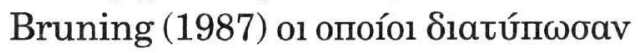

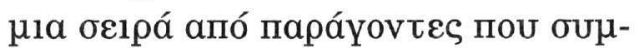

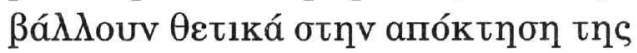

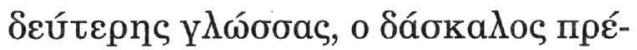

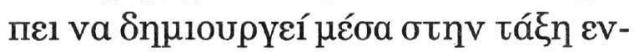

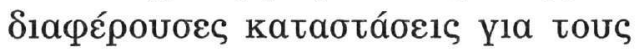
$\mu a \theta$ †

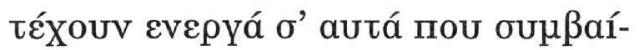

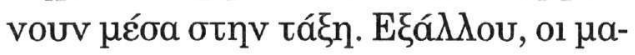

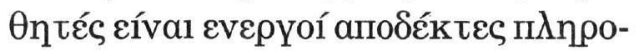

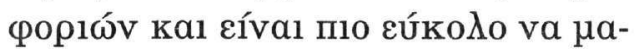

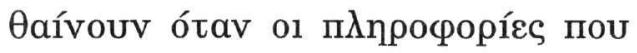

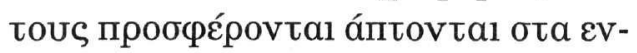

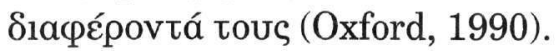

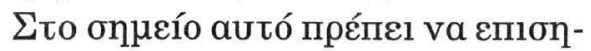

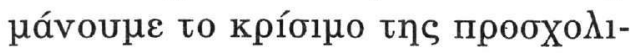

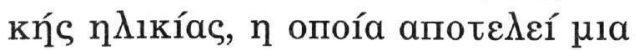

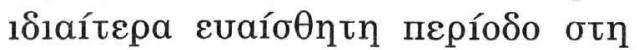

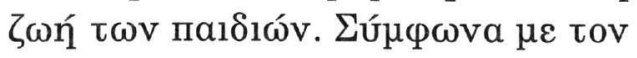

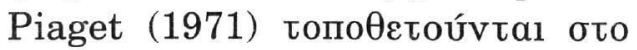

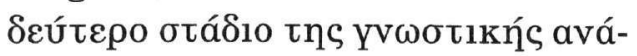

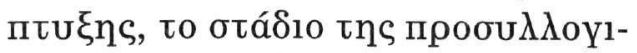

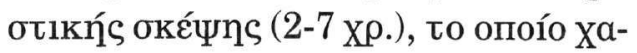

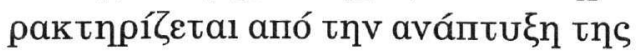

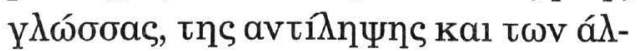

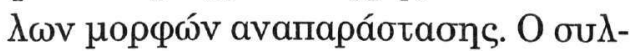

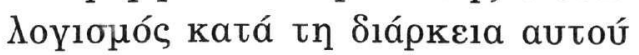

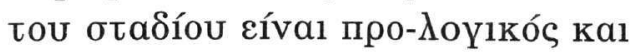

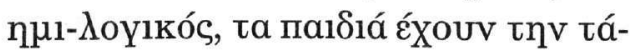

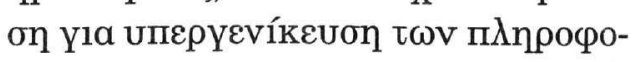

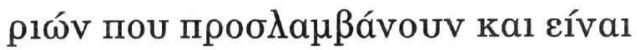

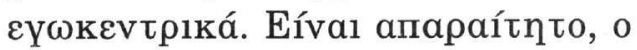

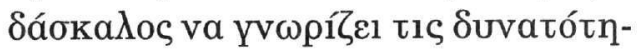

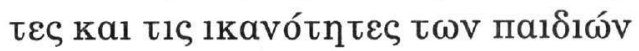

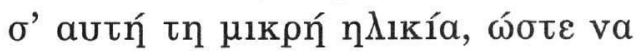

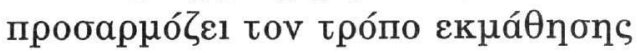
y $\lambda \omega \sigma \sigma a s$.

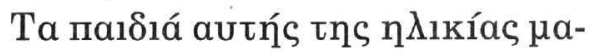

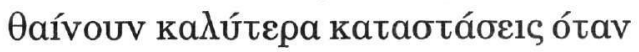

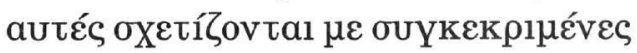

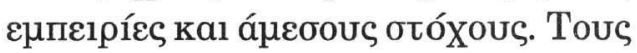

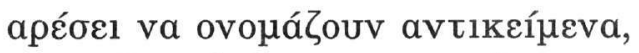

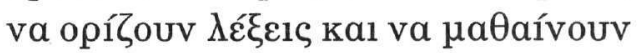

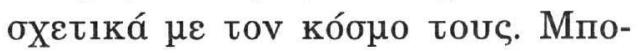

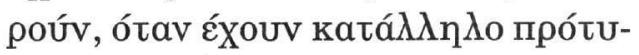

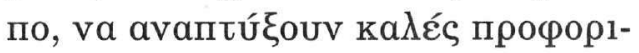

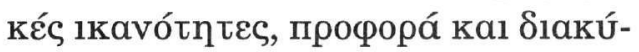

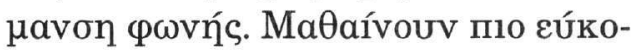

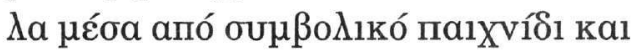

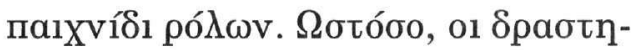

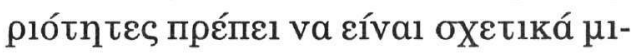

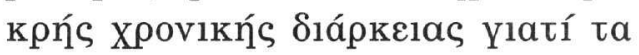

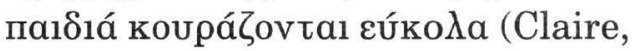
1988. Curtain, 1988). Н пореía точ

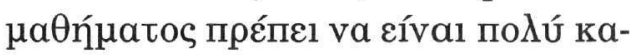

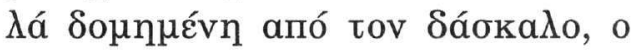

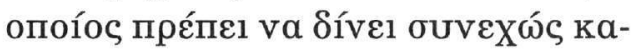

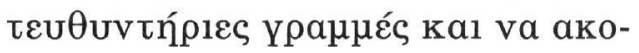

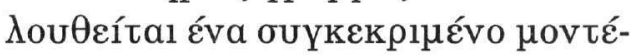

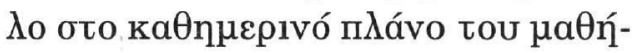
patoc (Spaulding, 1992).

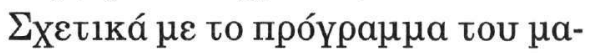

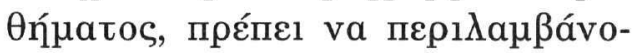

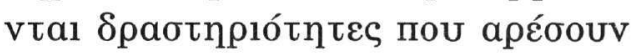

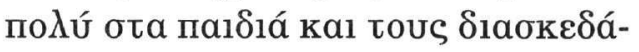

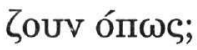

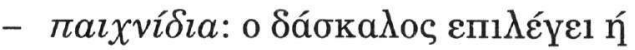

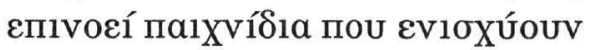

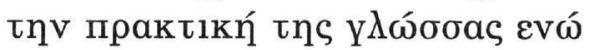

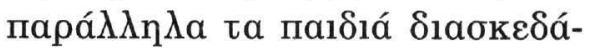

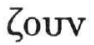

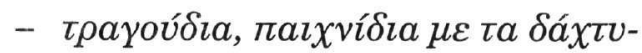




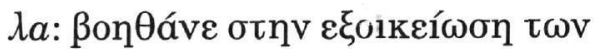

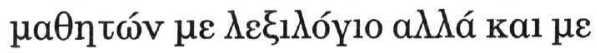

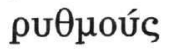

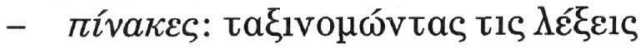

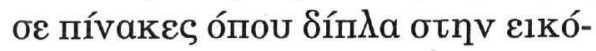

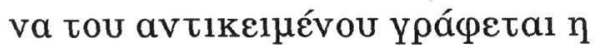

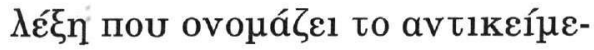

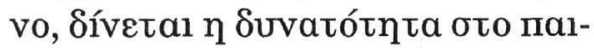

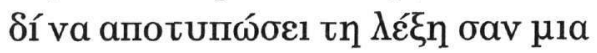

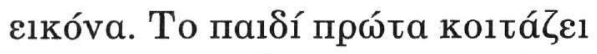

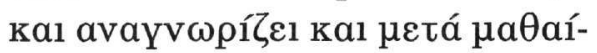
vع1 va $\mu 1 \lambda$ á́ı (Berger, 1986).

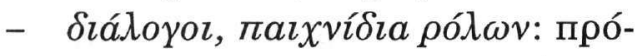

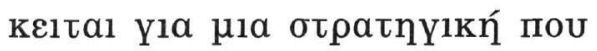

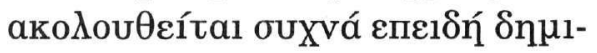

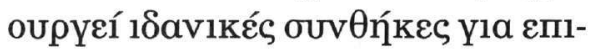

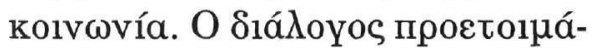

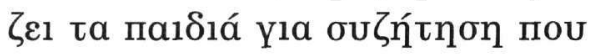

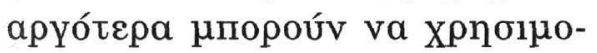

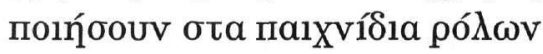

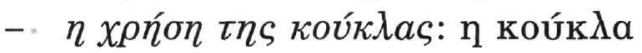

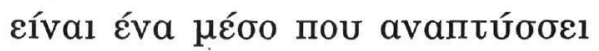

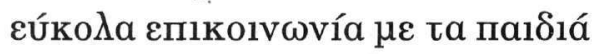

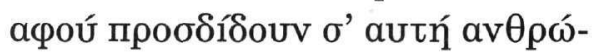

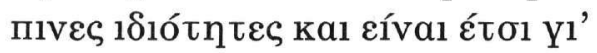

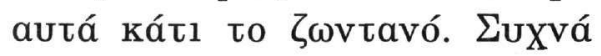

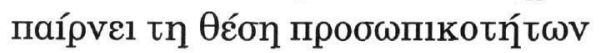

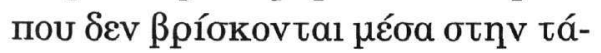

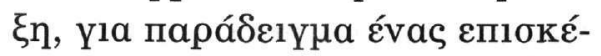

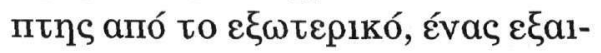

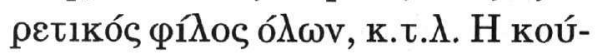

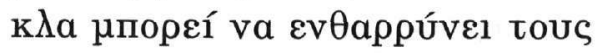

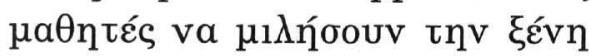

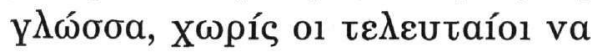

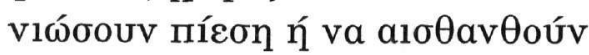

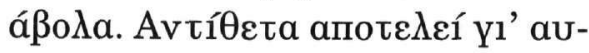

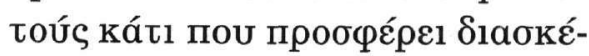

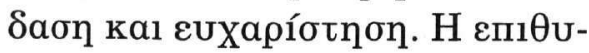

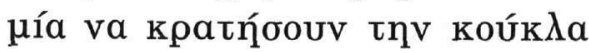


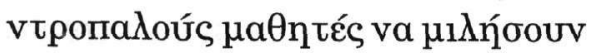

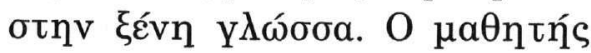

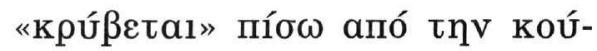

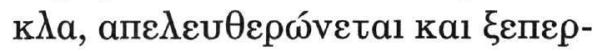

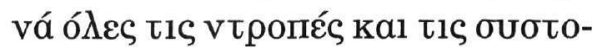
גéৎ tou.

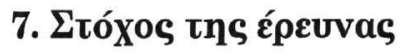

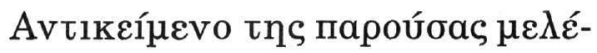

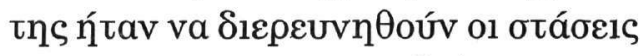

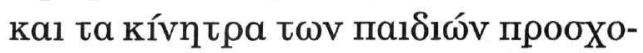

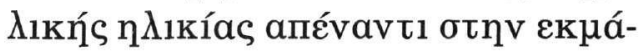

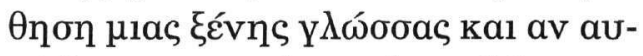

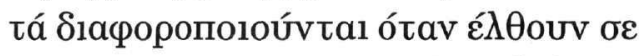

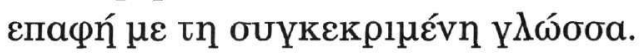

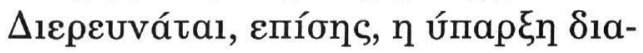


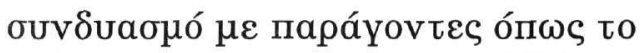

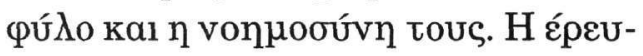

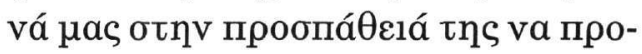

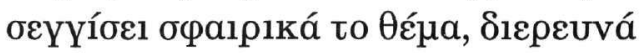

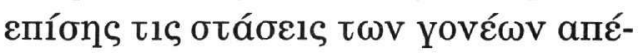

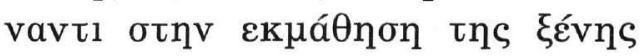

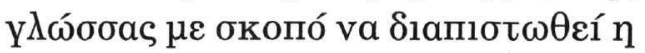

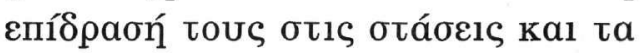

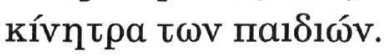

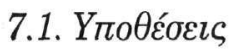

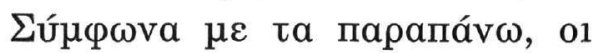

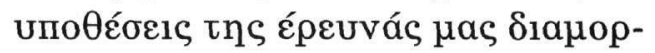

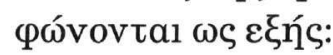

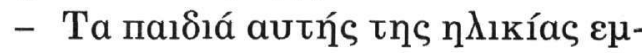

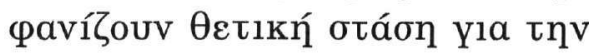

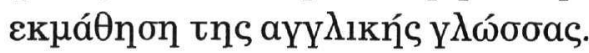

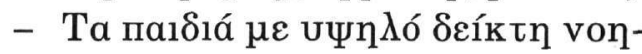

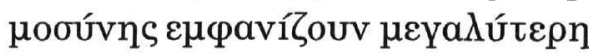

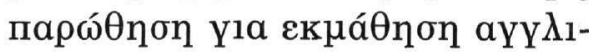
кńs Y $\lambda \omega \sigma \sigma a \varsigma$

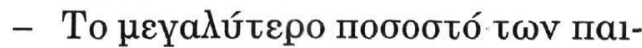

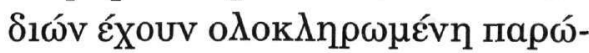

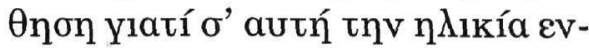

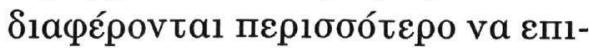




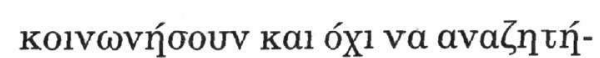

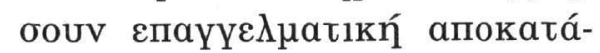

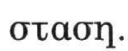

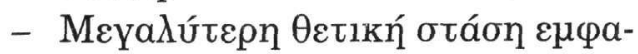

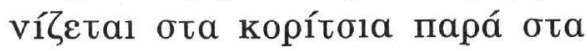
ayópla

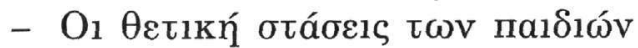

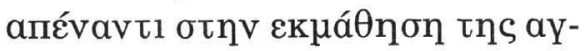

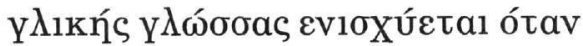

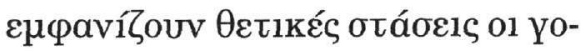
veíc tous.

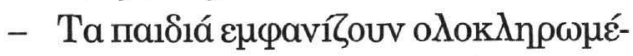

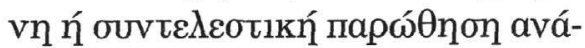

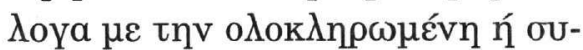

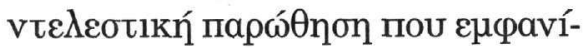

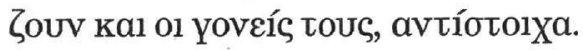

\section{2. $M \varepsilon ́ \theta 0 \delta o \varsigma$}

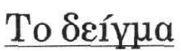

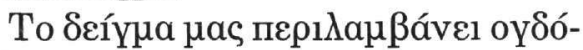

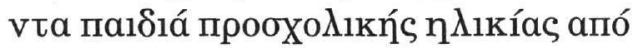

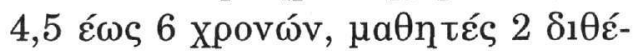

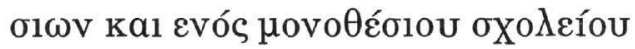

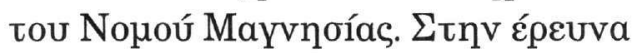

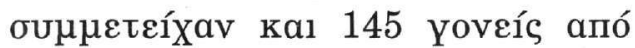

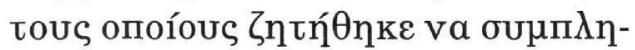

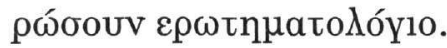

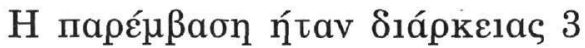

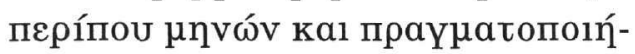

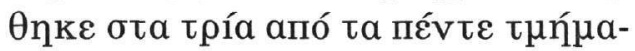

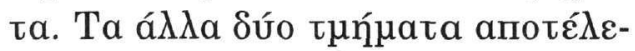

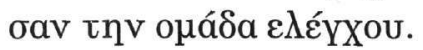

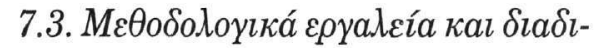
кaбía

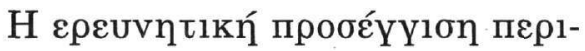

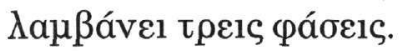

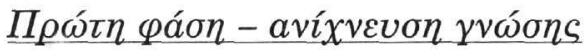

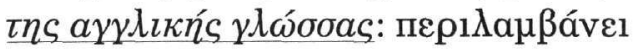

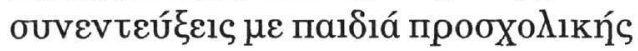

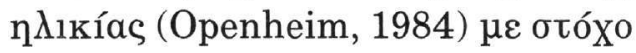
va

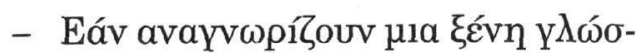
$\sigma a$

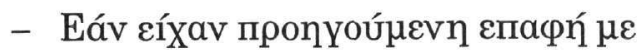

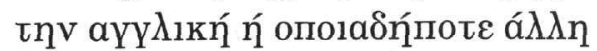

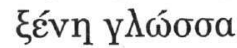

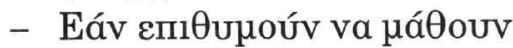

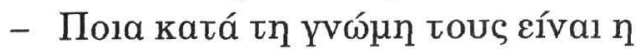

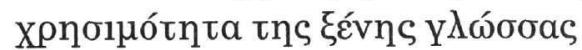

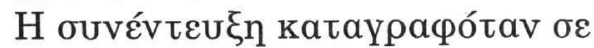

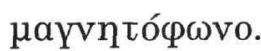

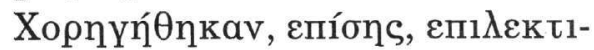

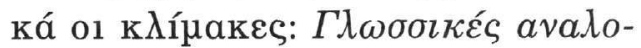

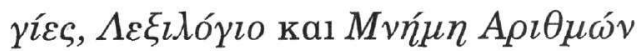

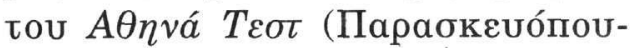

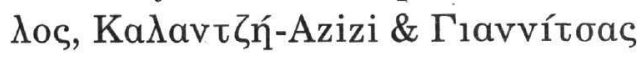

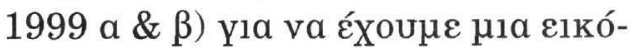

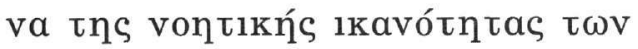

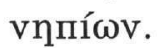

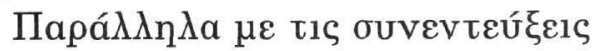

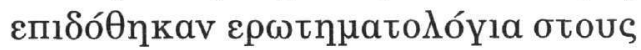

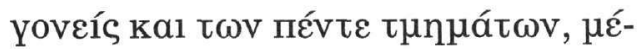

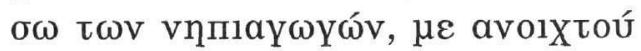

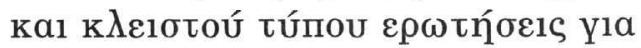

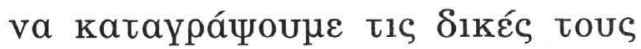

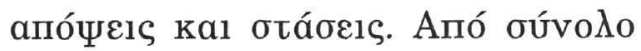

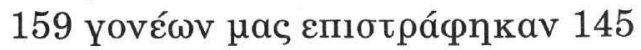

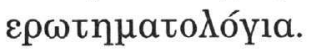

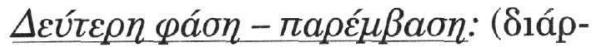

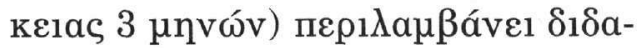

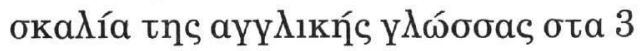

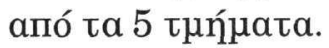

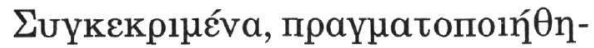

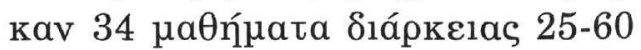

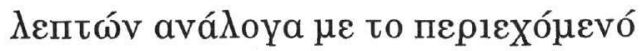

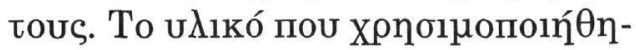

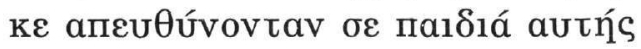

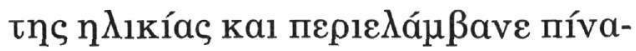
kєৎ ava

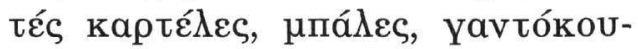

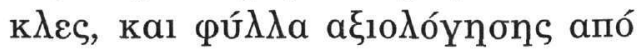

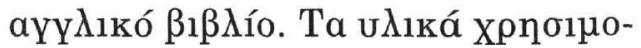

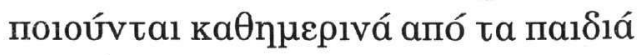




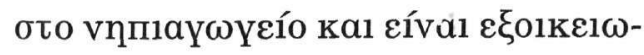
$\mu \varepsilon ́ v a$ p' autá.

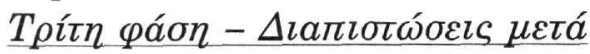

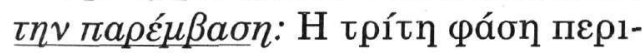

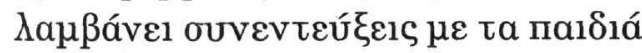

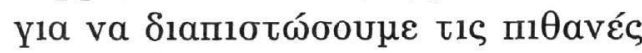

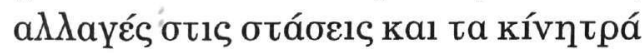

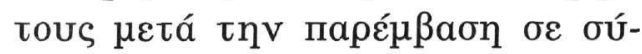

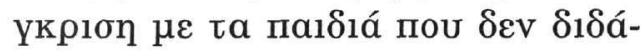

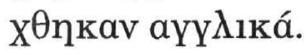

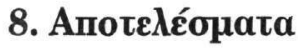

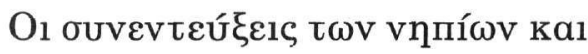
ol avoixtés ع

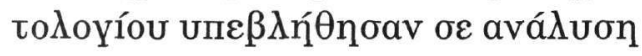

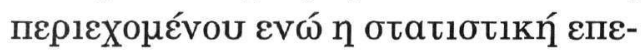

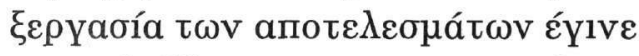

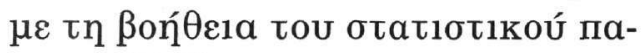
кétou SPSS.

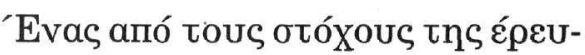

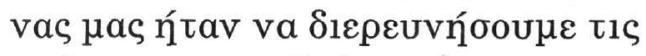

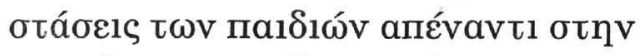

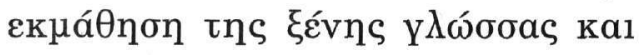

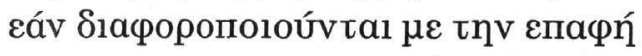

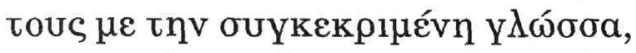

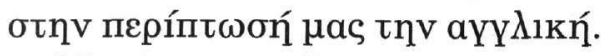

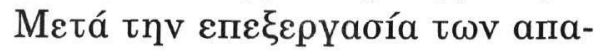

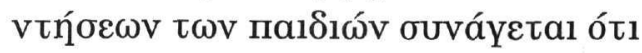

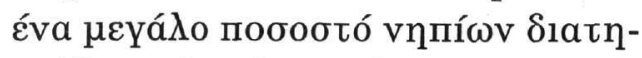

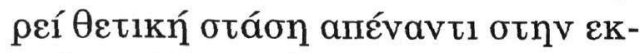

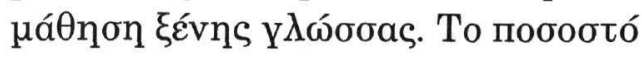

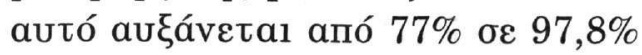

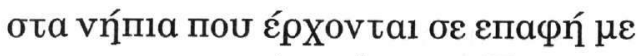

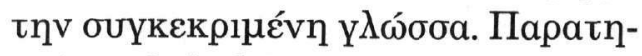

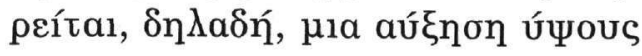

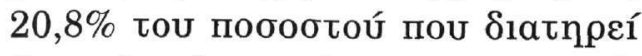

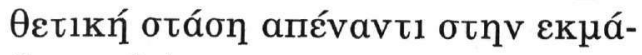
$\theta \eta \sigma \eta ~ Y \Lambda \omega \sigma \sigma \alpha \varsigma$.

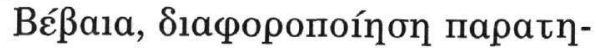

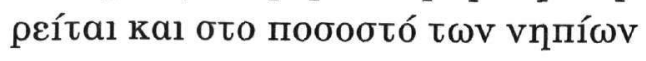

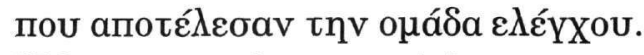

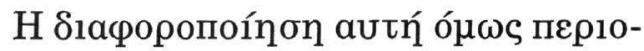

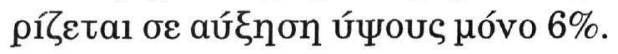

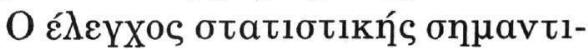

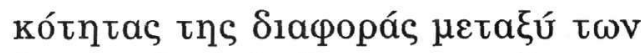

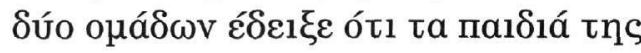

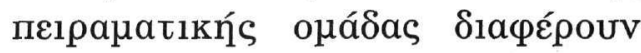

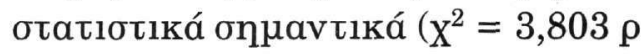

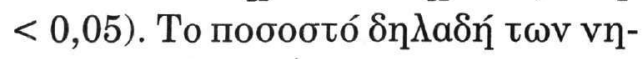

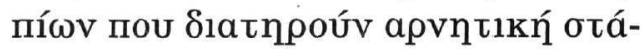

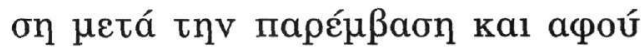

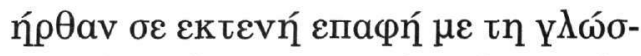

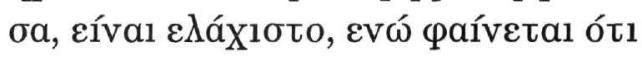

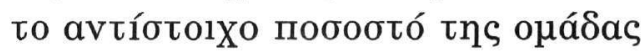

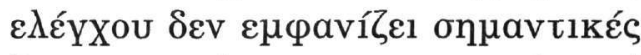

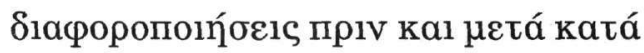

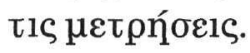

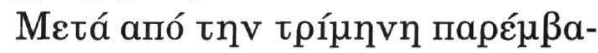

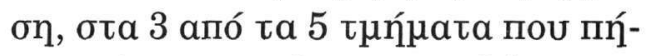

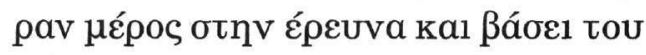

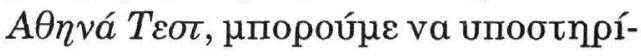

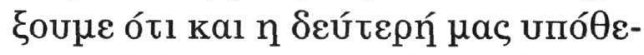

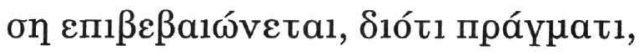

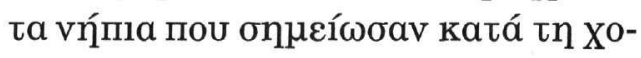

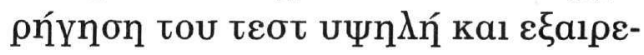

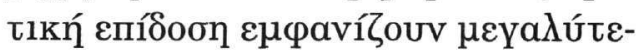

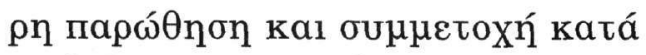

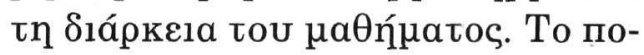

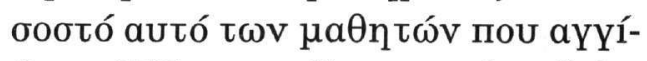

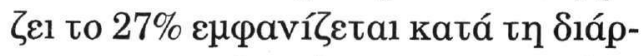

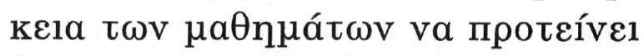

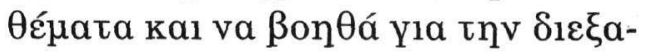

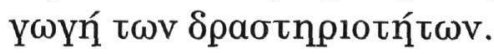

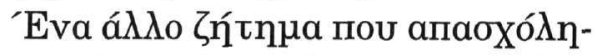

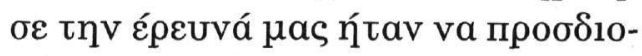

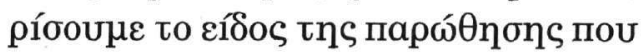

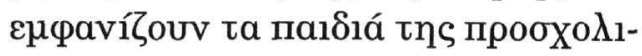

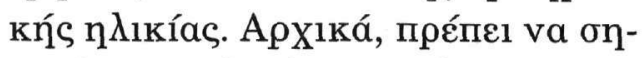

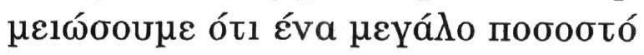

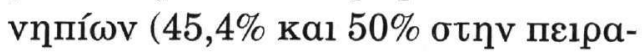

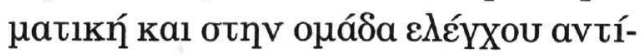

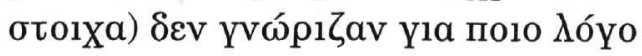




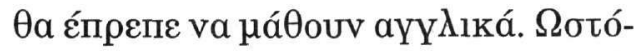

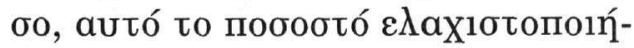

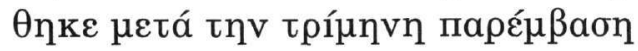

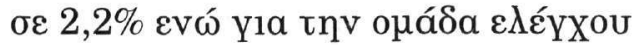

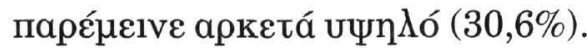

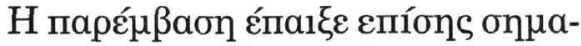

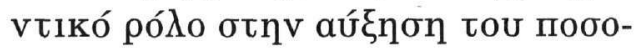

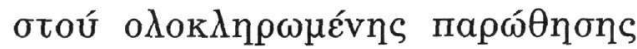

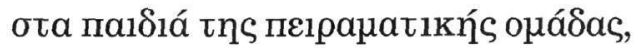
a

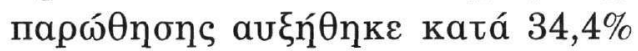

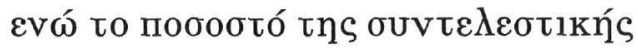
нóvo $8,8 \%$.

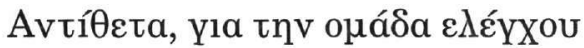

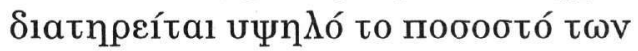

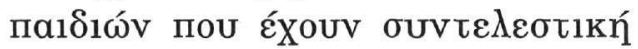

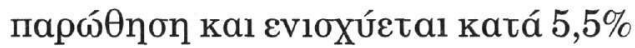

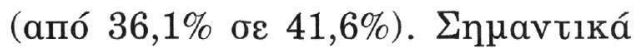

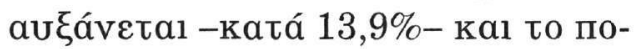

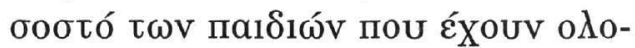

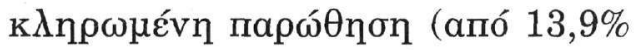

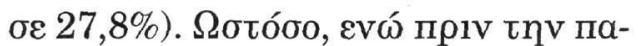

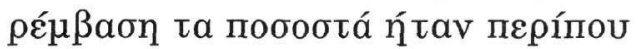

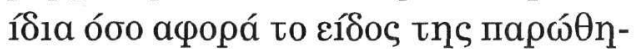
on

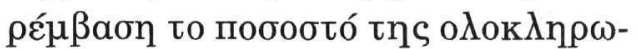

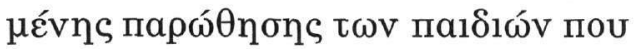

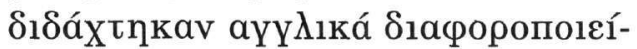

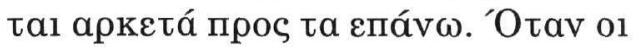

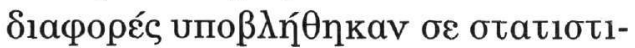

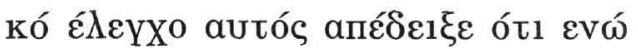

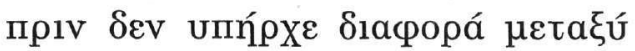

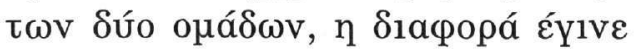

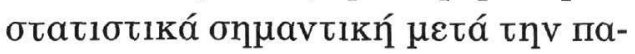

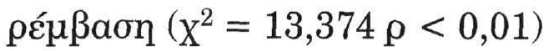

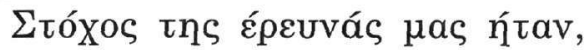

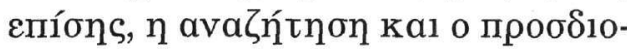

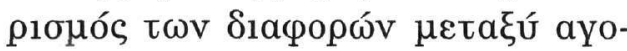

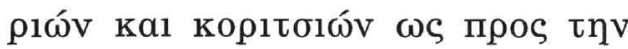

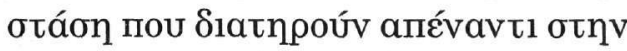

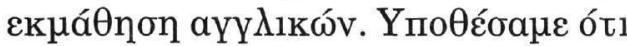

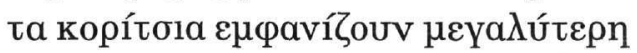

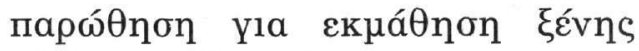

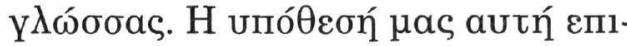

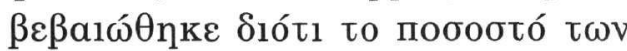

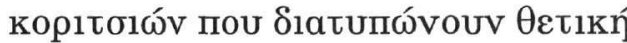

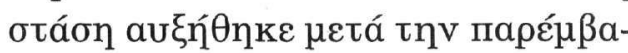

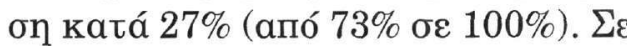

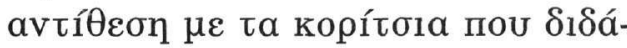
X

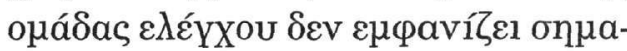

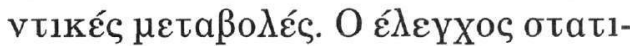

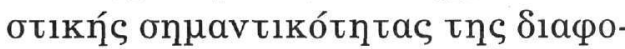

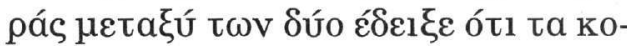

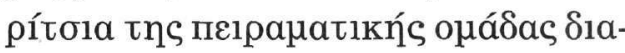

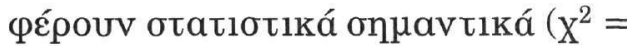
$4,823 \rho<0,05$ ). Ta ayópia $\delta \varepsilon v$ on-

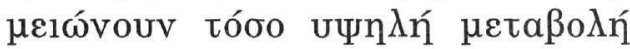

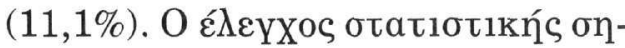

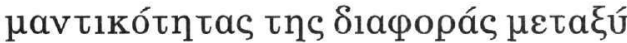

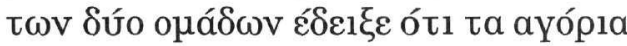

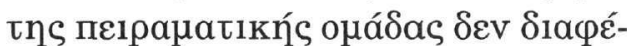

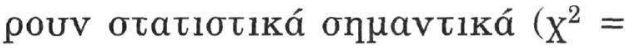
$0,055 \rho<0,814)$.

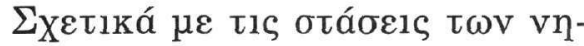

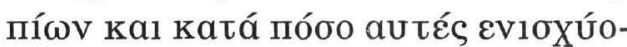
vtal ótav epqaví̉ouv kal ol yoveís

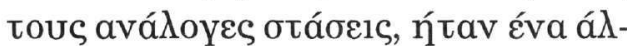

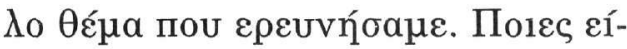

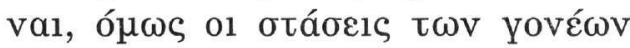

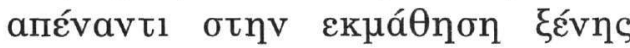
y

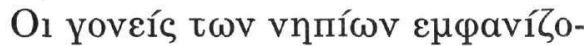

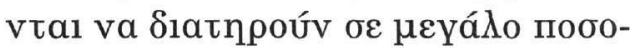

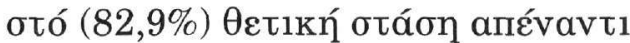

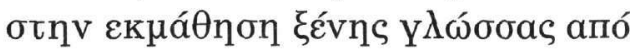

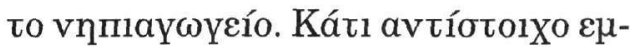
qaví̉etal kal yla tous yoveís tns

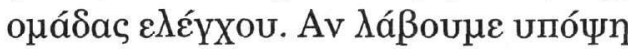

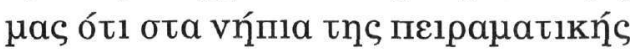

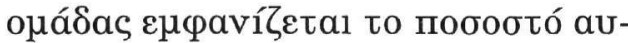

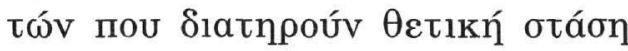

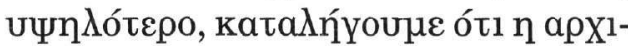

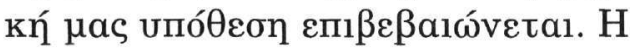




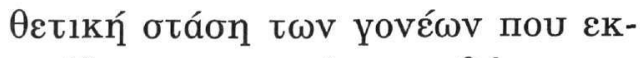

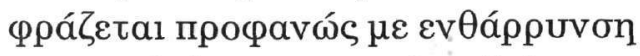

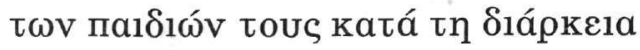

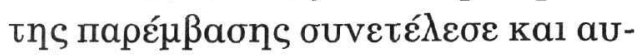

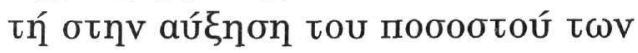

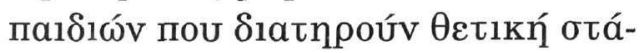

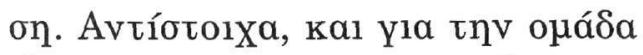

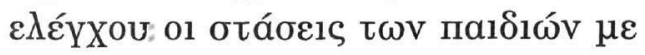

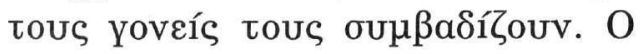

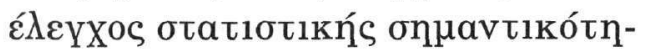

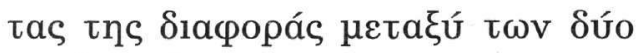

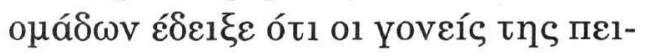

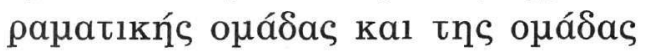

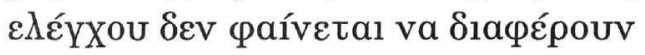

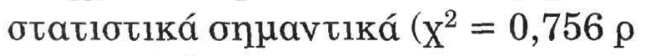
$<0,385 / X^{2}=1,497 \rho<0,221$ Y 1a 七ov

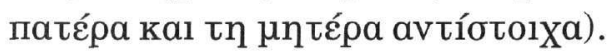

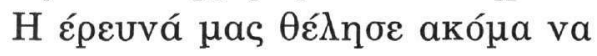

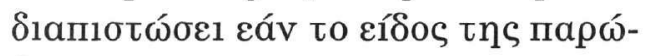

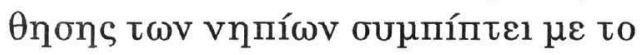

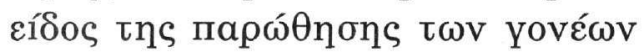

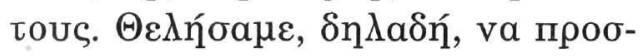

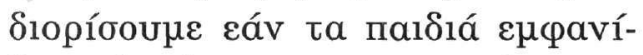

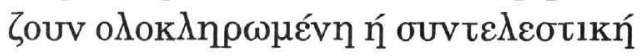

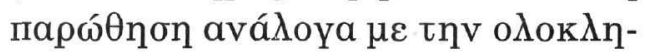

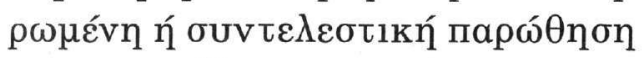

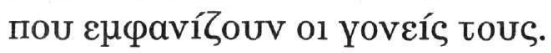

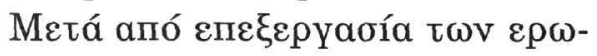

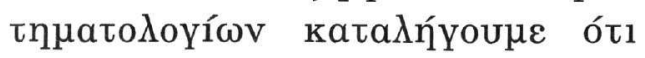

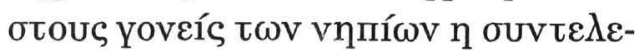

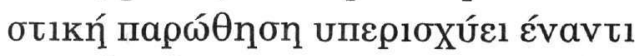

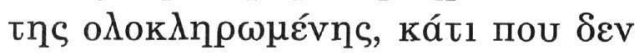

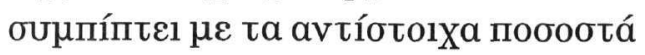

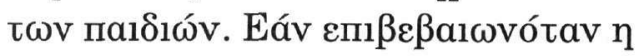

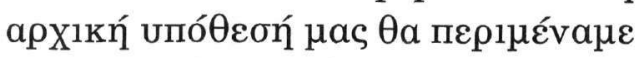

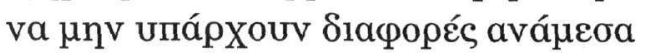

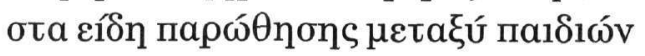

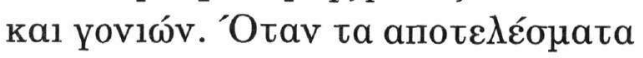

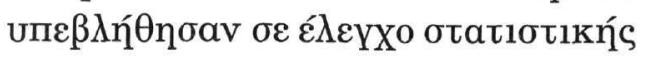

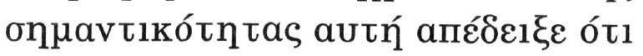

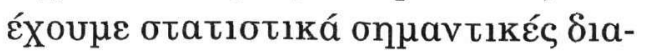
甲оре́ৎ $\left(\mathrm{X}^{2}=1,921 \rho<0,05 \mathrm{X}^{2}=\right.$

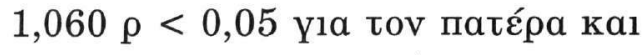

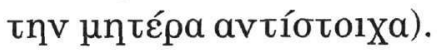

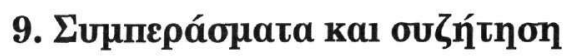

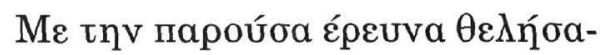

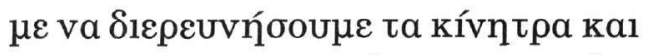

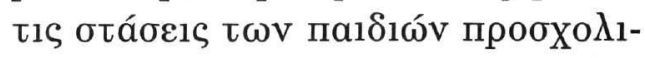

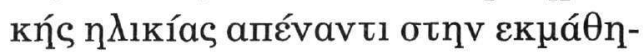

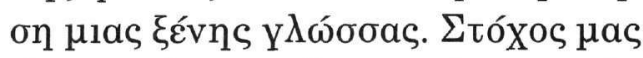

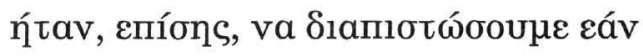

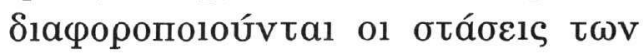

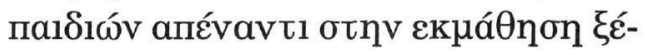

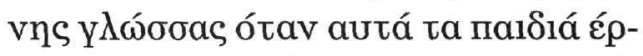

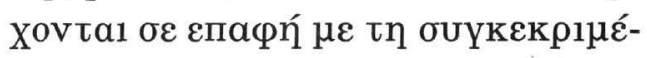

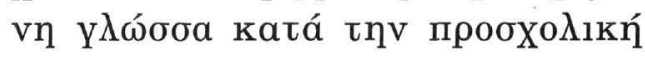

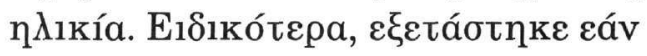

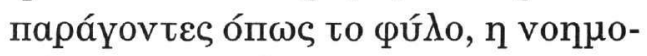

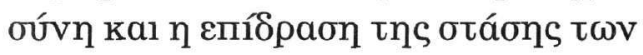

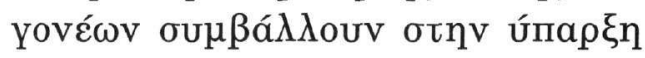

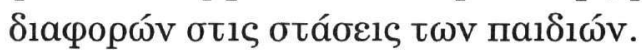

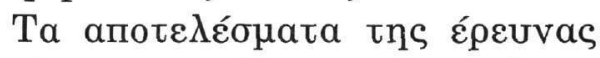

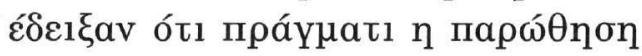

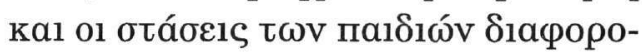

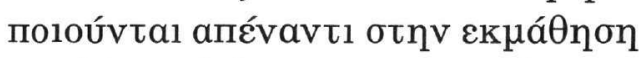

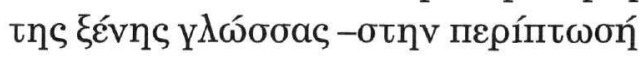

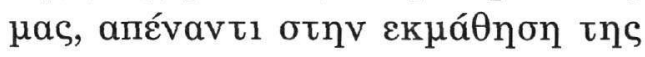

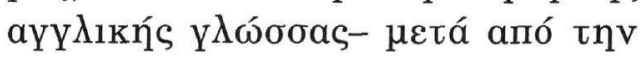

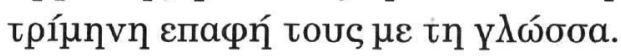

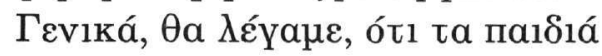

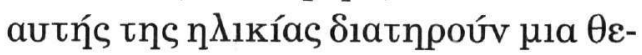

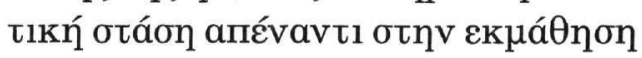

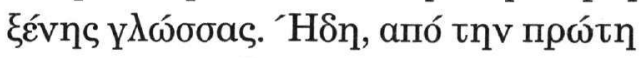

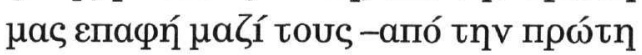

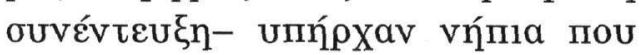

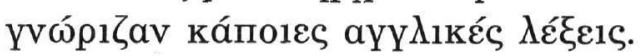

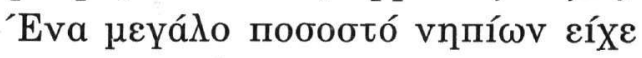

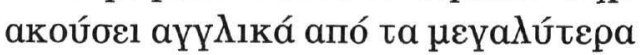

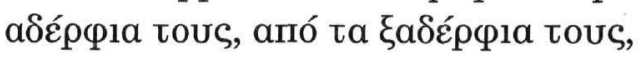

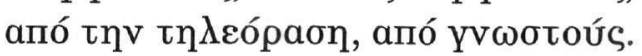

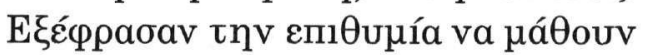




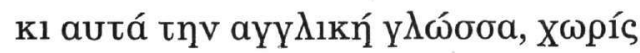

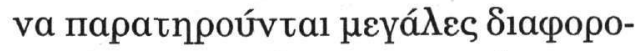

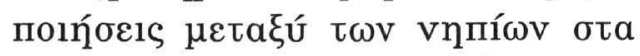

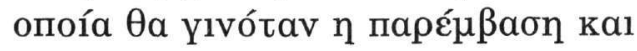

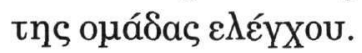

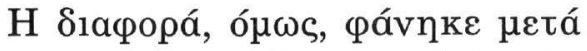

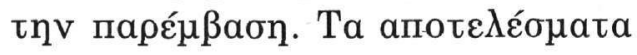

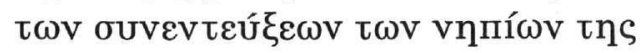

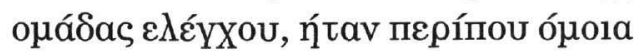

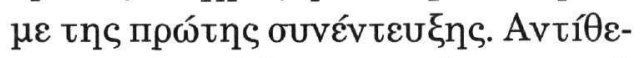

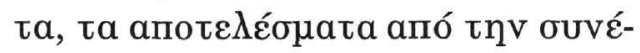

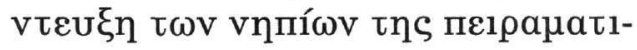

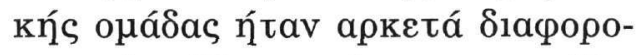

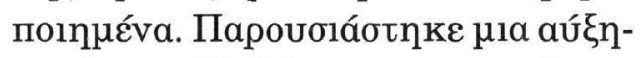

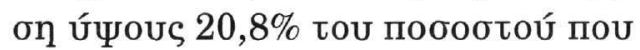

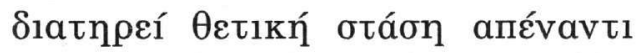

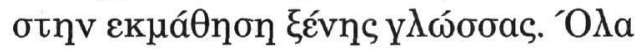

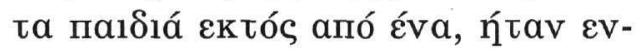

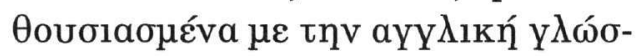

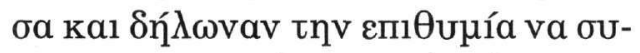

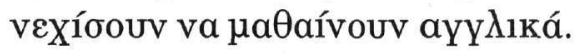

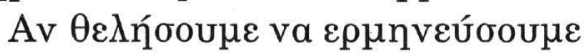

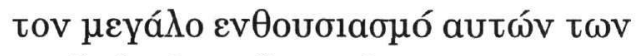

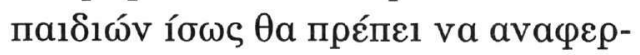

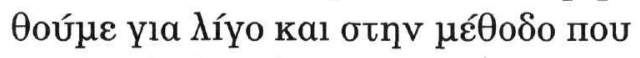

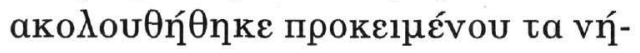

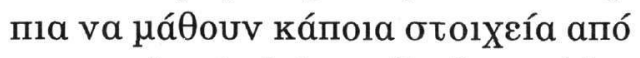

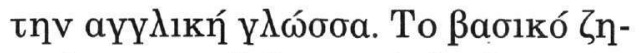

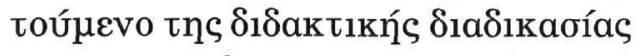

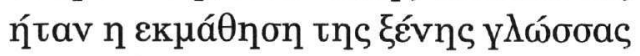

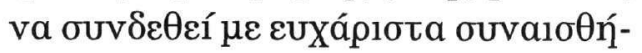

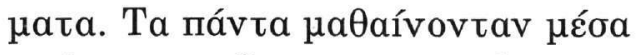

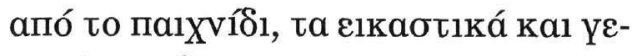

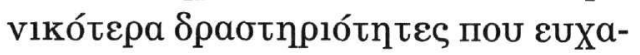

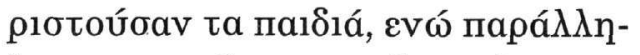

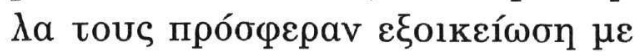

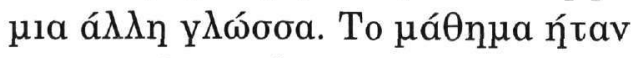

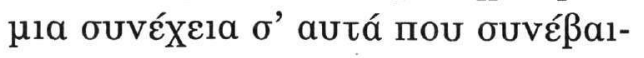

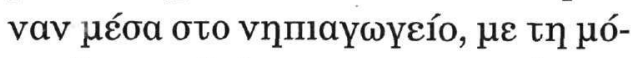

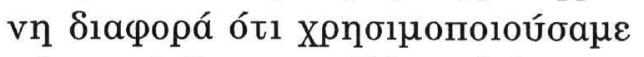

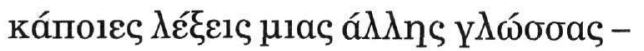

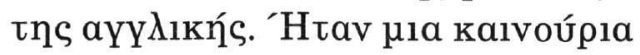

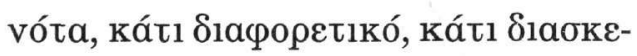

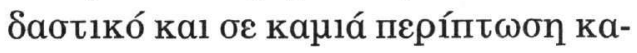
tavaykaбtıkó. Ta паıઠı́á Xaípovtav

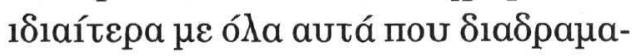

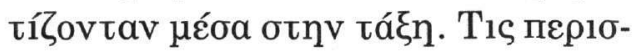

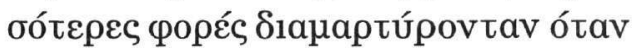

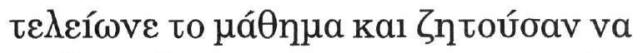

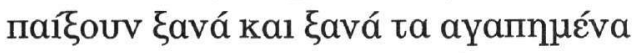

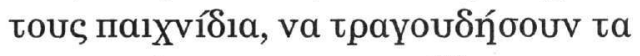

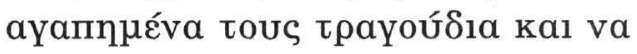

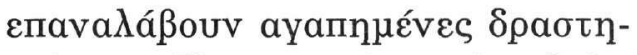

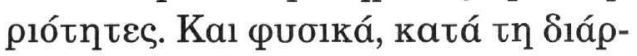

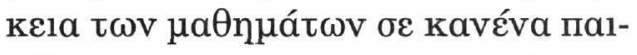

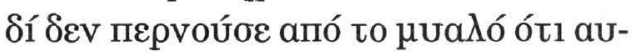

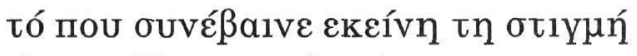

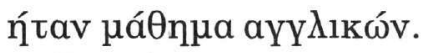

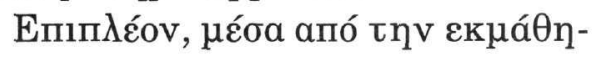

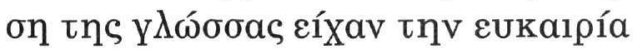

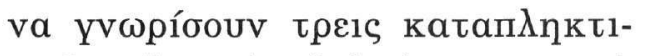

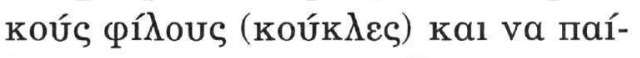

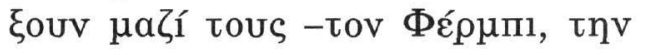

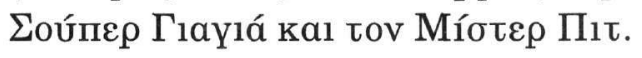

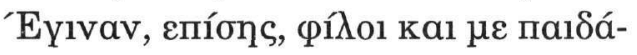

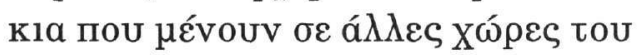

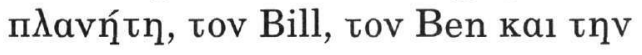

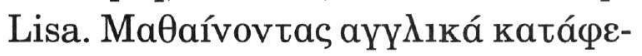

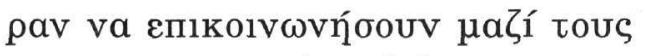

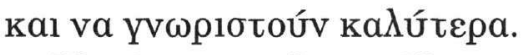

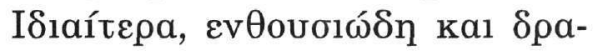

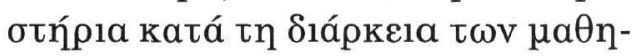

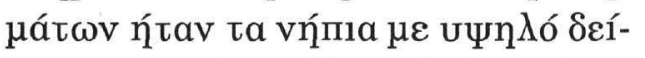

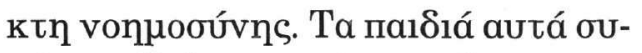

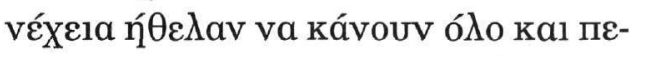

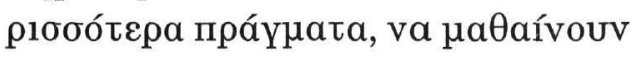

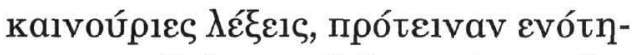

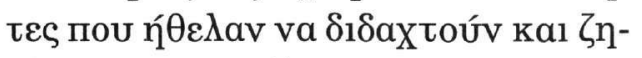

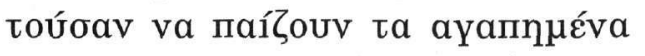

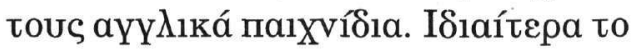
«Stand up - Sit down» (пalXvíס1)

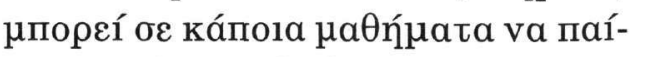
Х

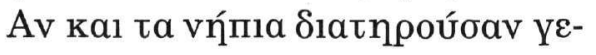




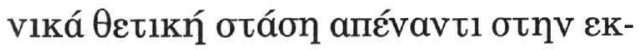

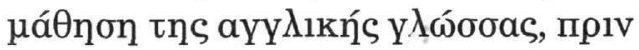

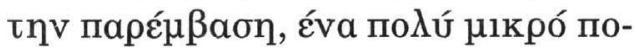

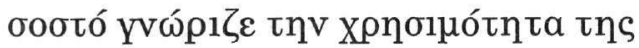
aүY

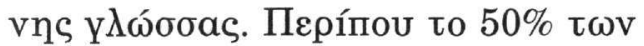

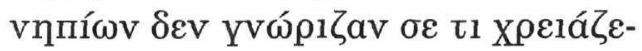

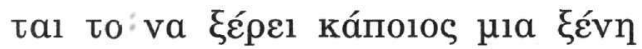

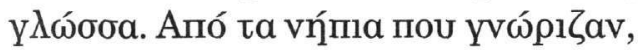

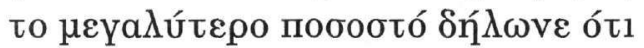

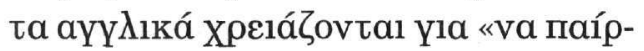

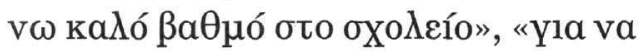

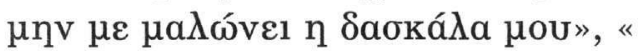

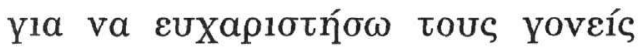

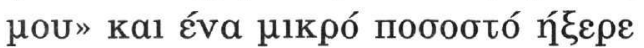

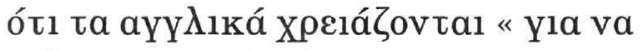

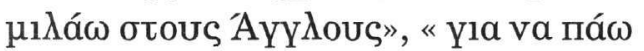

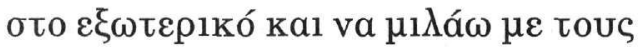

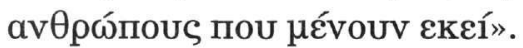

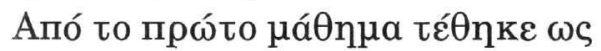

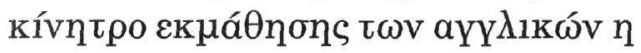

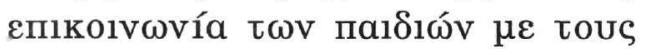

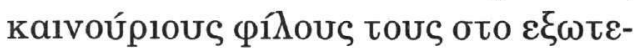

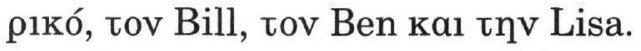

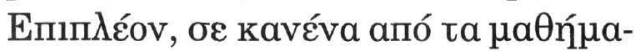

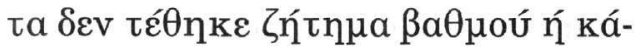

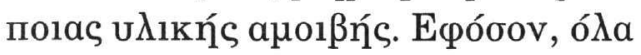

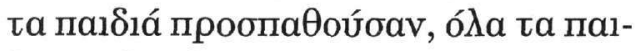

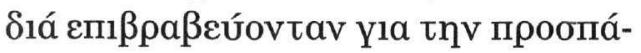

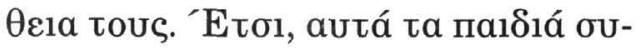

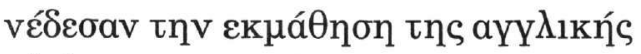

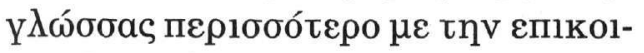

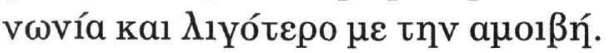

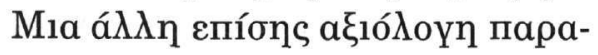

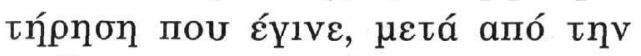

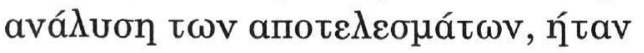

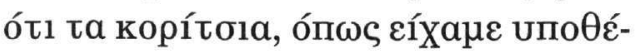

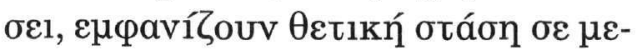
yaגútepo побоб

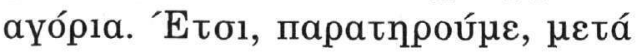

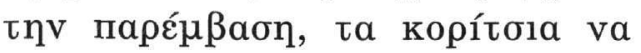

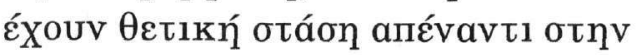

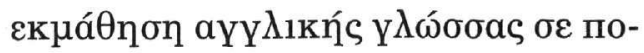

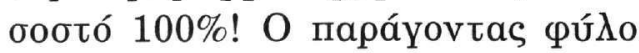

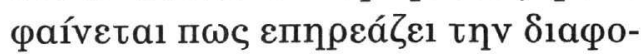

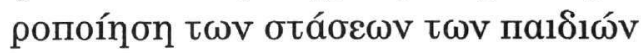

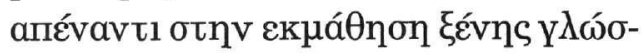

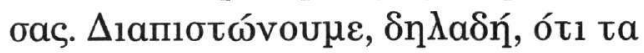

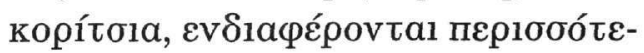

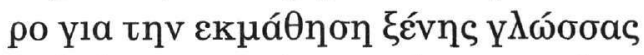

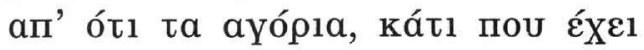

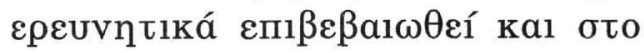
парєגӨóv (Gardner, 1985).

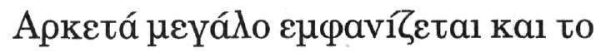

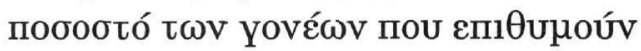

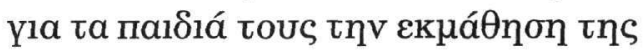

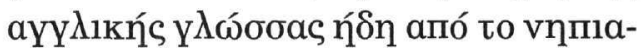

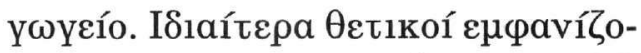

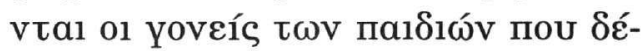

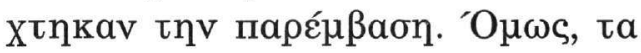

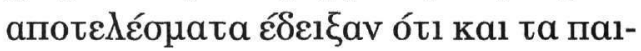

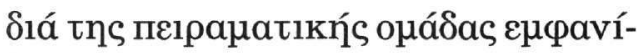

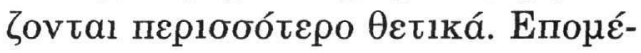

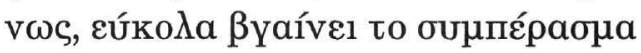

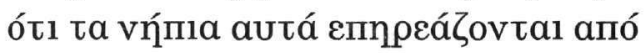

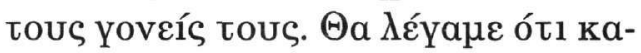

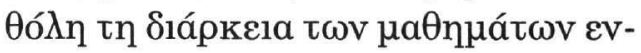

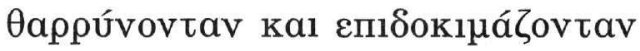

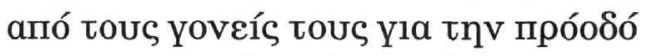

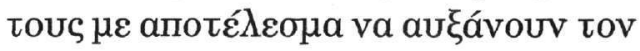

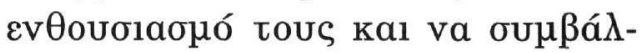

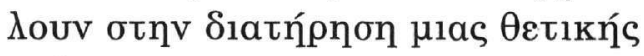
otáoņs.

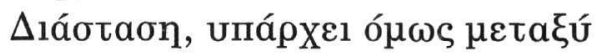

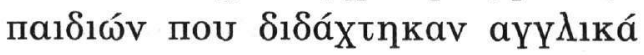

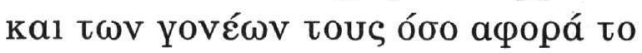

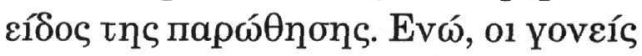

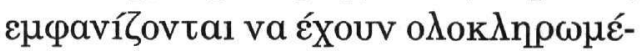

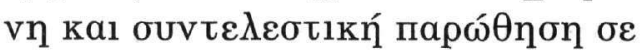

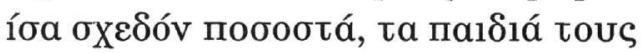

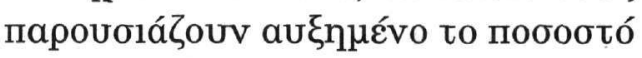

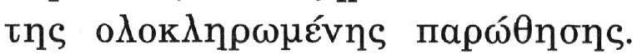

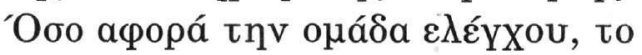

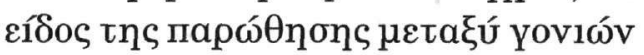




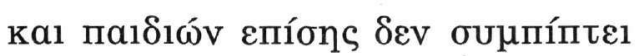

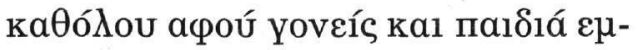

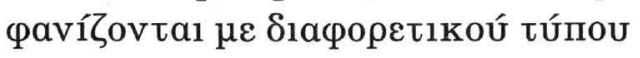

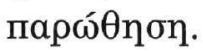

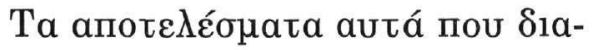

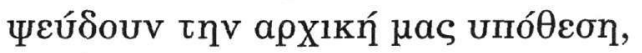

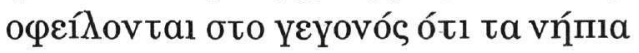

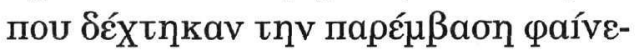

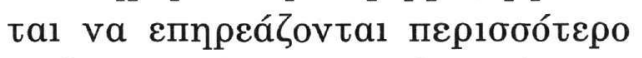

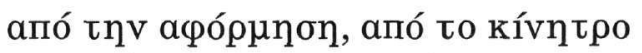

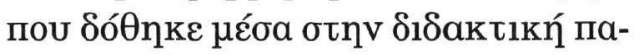

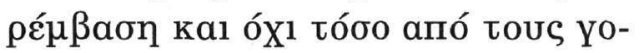

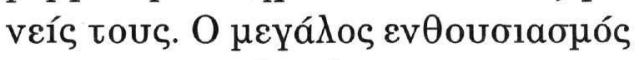

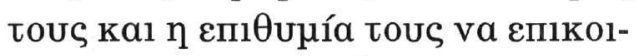

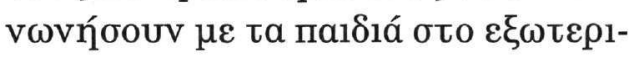

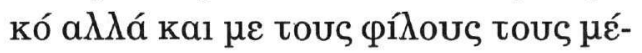

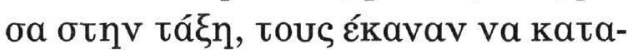

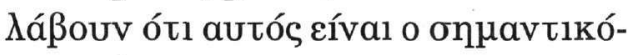

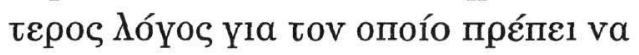

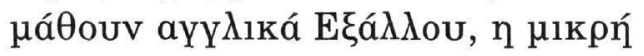

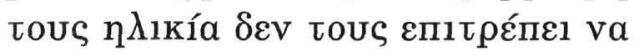

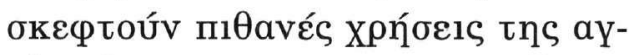

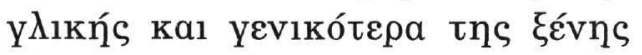

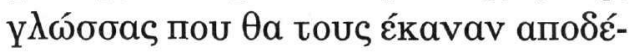

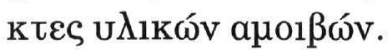

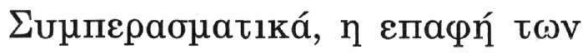

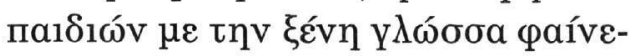

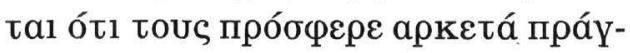

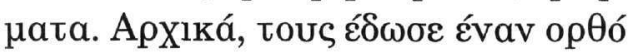

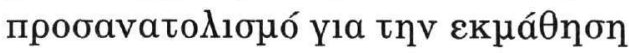

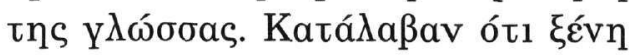

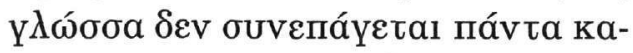

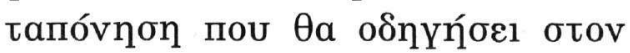

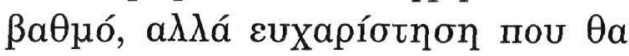

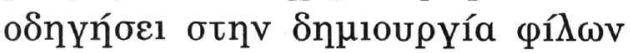

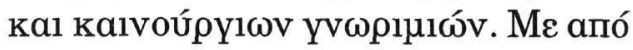

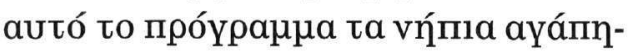

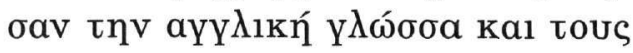

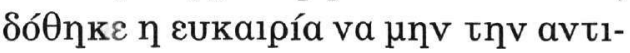

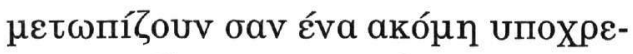

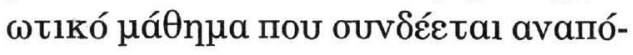

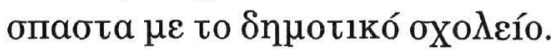

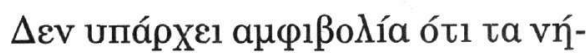

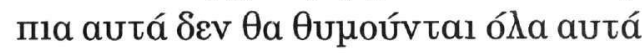

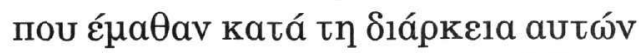

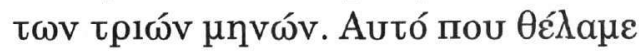

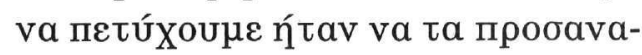

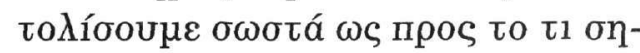

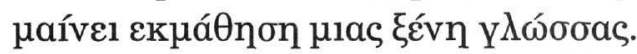

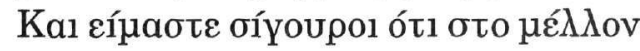

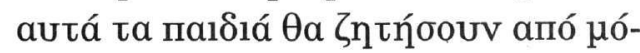

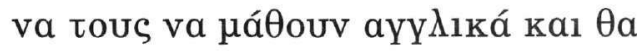
volẃ $\theta$ ouv ótı autá nou kávouv tous

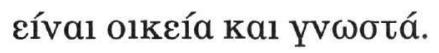

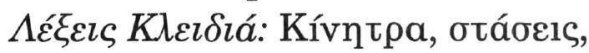

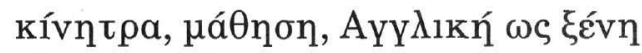
y $\lambda \omega \sigma \sigma a$, vímı

\section{Bißגıүрачía}

Berger, J. (1986). H ع1kóva ka1 to

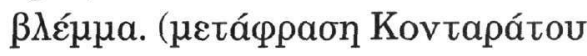

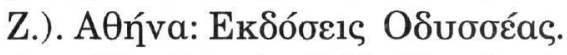

Claire, El. (1988). ESL Teacher's Activities kit. New Jersey: Prentice Hall.

Clement, R. \& Kruidenier, B.G. (1985). Aptitude, Attitude and Motivation in Second Language Proficiency: A Test of Clement's Model. Journal of Language and Social Psychology, 4, 21-37.

Curtain, H. (1988). Languages and Children, making the match. London: Addison Wesley Publishing Company.

Dweck, C., (1986). Self - Theories: Their role in motivation, personality and development. New York: Taylor \& Francis Group.

Gardner, R. (1985). Social Psychology and Second Language Learning: The Role of Attitudes and Moti- 
vation. London: Edward Arnold. Gardner, R. (1995). Expanding the Motivation Construct in Language Learning. Modern Language Learning, 79, 505-517.

Gardner, R. \& Lambert, W. (1972). Attitudes and Motivation in Second Language Learning. Rowley, MA: Newbury House.

Glover, J.A. \& Bruning, R. (1987). Educational Psychology: Principles and Applications. 2nd ed. Boston: Little, Brown and Company.

Lambert, W.E., \& Klineberg, O. (1967). Children's Views of Foreign people. New York: Appleton - Century - Crofts.

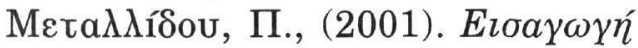

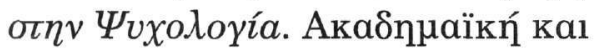

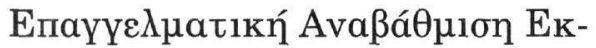

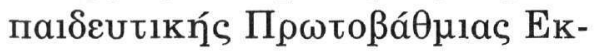

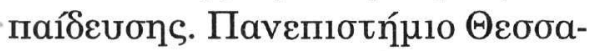
Nías. Bódos.

Oppenheim, A.N. (1984). Questionnaire Design and Attitude Measurement. London. Biddles Ltd.

Oxford, R. (1990). Language Learning Strategies: What Every Teacher Should Know. New York: Newbury House/ Harper \& Row.

Oxford, R. \& Shearin, J. (1994). Language Learning Motivation: Expanding the theoretical framework. The Modern Language Journal, 78, 12-28.

Паппа́, Е. \& Zачєıропоú (2000). O pódoc $\tau \omega \mathrm{v}$ Kıví $\rho \omega \mathrm{v}$

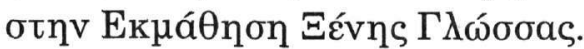

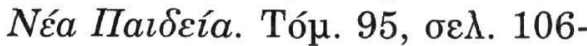
119.

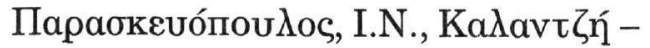

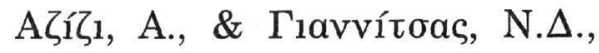

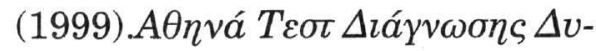

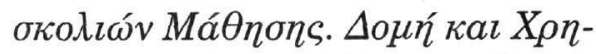

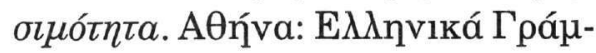
pata.

Парабкєuónouגos, I.N., Kaגavtלń-

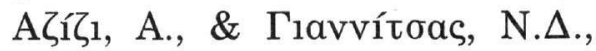

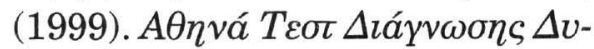

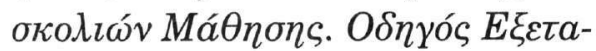

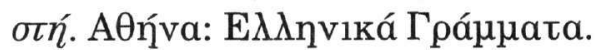
Piaget, J. (1971). The Theory of Stage Cognitive Development. In D. R. Green, M. P. Ford and G. B. Flamer (Eds.) Measurement and Piaget. New York: McGraw-Hill. Pintrich, P.R. (1999). The Role of Motivation in Promoting and Sustaining Self-regulated Learning. International Journal of Educational Research, 31, 459-470.

Skehan, P. (1989). Individual Differences in Second-Language Learning. London: Edward Arnold. Spaulding, L.C. (1992). Motivation in the Classroom. McGraw-Hill, Inc.

Spolsky, B. (1985). Formulating a Theory of Second Language Learning. Studies in Secon Language Acquisition, 7, 269-288.

\section{Abstract}

The advantages of early learning of a foreign language have been a popular subject of scientific research. Recent findings argue that motivation and attitudes play an important role in foreign language learning and that motivation of children attenuates with age. The 
aim of the present study was to investigate the motivation and attitudes of Greek kindergarten children towards learning English as a foreign language and also to study if early familiarization with the language has an effect on their attitudes and motivation later on. The impact of parental attitudes on children's motivation was also investigated. Eighty kindergarten children and 146 parents took part in the study. Our findings show that young children are positively motivated and have an equally positive attitude toward English language learning, which strengthens significantly when they-and especially the girls-come in contact with the language in their schools early.

\section{$\Delta 1 \varepsilon u ́ \theta u v o n$ Emikoivwvías}

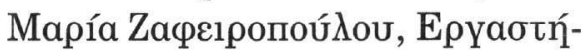

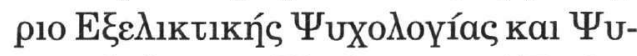

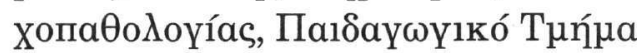

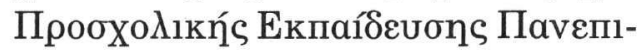

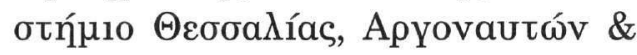
$\Phi_{1} \lambda \varepsilon \lambda \lambda \eta \underline{v \omega}$. 38221, Bódoc.

$\operatorname{T\eta } \Lambda$.Fax: $042174736 \& 018075978$.

E-mail: mzafirop@uth.gr \& zafirop@groovy.gr 